\title{
Optimal regularity in the obstacle problem for Kolmogorov operators related to American Asian options
}

\author{
Marie Frentz* \\ Department of Mathematics, Umeå University \\ S-90187 Umeå, Sweden \\ Kaj Nyström ${ }^{\dagger}$ \\ Department of Mathematics, Umeå University \\ S-90187 Umeå, Sweden \\ Andrea Pascucci $\ddagger$ \\ Dipartimento di Matematica, Università di Bologna \\ Piazza di Porta S. Donato 5, 40126 Bologna, Italy \\ Sergio Polidoro $\$$ \\ Dipartimento di Matematica Pura ed Applicata, \\ Università di Modena e Reggio Emilia \\ Via Campi, 213/b 41100 Modena, Italy
}

September 15, 2008

\begin{abstract}
In this paper we prove optimal interior regularity for solutions to the obstacle problem for a class of second order differential operators of Kolmogorov type. We treat smooth obstacles as well as non-smooth obstacles. All our proofs follow the same line of thought and are based on blow-ups, compactness, barriers and arguments by contradiction. This problem arises in financial mathematics, when considering path-dependent derivative contracts with the early exercise feature.
\end{abstract}

2000 Mathematics Subject classification.

Keywords and phrases: operator of Kolmogorov type, obstacle problem, hypoelliptic, regularity, blow-up.

*email: marie.frentz@math.umu.se

†email: kaj.nystrom@math.umu.se

†email: pascucci@dm.unibo.it

$\S$ email: sergio.polidoro@unimore.it 


\section{Introduction}

This paper is devoted to the obstacle problem for a class of second order differential operators of Kolmogorov type of the form

$$
L=\sum_{i, j=1}^{m} a_{i j}(x, t) \partial_{x_{i} x_{j}}+\sum_{i=1}^{m} b_{i}(x, t) \partial_{x_{i}}+\sum_{i, j=1}^{N} b_{i j} x_{i} \partial_{x_{j}}-\partial_{t}
$$

where $(x, t) \in \mathbb{R}^{N+1}, m$ is a positive integer satisfying $m \leq N$, the functions $\left\{a_{i j}(\cdot, \cdot)\right\}$ and $\left\{b_{i}(\cdot, \cdot)\right\}$ are continuous and bounded and the matrix $B=\left\{b_{i j}\right\}$ is a matrix of constant real numbers. Let $\Omega \subset \mathbb{R}^{N+1}$ be an open subset, let $\partial_{P} \Omega$ denote the parabolic boundary of $\Omega$, let $g, f, \psi: \bar{\Omega} \rightarrow \mathbb{R}$ be such that $g \geq \psi$ on $\bar{\Omega}$ and assume that $g, f, \psi$ are continuous and bounded on $\bar{\Omega}$. We consider the following problem for the operator $L$,

$$
\begin{cases}\max \{L u(x, t)-f(x, t), \psi(x, t)-u(x, t)\}=0, & \text { in } \Omega, \\ u(x, t)=g(x, t), & \text { on } \partial_{P} \Omega .\end{cases}
$$

The structural assumptions imposed on the operator $L$, which will imply that $L$ is a hypoelliptic ultraparabolic operator of Kolmogorov type, as well as the regularity assumptions on $a_{i j}, b_{i}, f$, $\psi$ and $g$ will be defined and discussed below. We note that in case $m=N$ the assumptions we impose imply that the operator $L$ is uniformly elliptic-parabolic while if $m<N$ then the operator $L$ is degenerate and not uniformly elliptic-parabolic. In particular, we are mainly interested in the case $m<N$. The problem in (1.2) represents the obstacle problem for the operator $L$ with obstacle $\psi$, boundary data $g$ and right hand side defined by $f$.

To motivate our study of the problem in (1.2) we note that obstacle problems are of fundamental importance in many applications in physics, biology and mathematical finance. In particular, one important problem in mathematical finance is that of determining the arbitrage free price of options of American type. More precisely, consider a financial model where the dynamics of the state variables is described by a $N$-dimensional diffusion process $X=\left(X_{t}^{x, t_{0}}\right)$ which is a solution to the stochastic differential equation

$$
d X_{t}^{x, t_{0}}=B X_{t}^{x, t_{0}}+\sigma\left(X_{t}^{x, t_{0}}, t\right) d W_{t}, X_{t_{0}}^{x, t_{0}}=x
$$

where $\left(x, t_{0}\right) \in \mathbb{R}^{N} \times[0, T]$ and $W=\left\{W_{t}\right\}$ denotes a $m$-dimensional Brownian motion, $m \leq N$. An American option with pay-off $\psi$ is a contract which gives the holder the right to receive a payment equal to $\psi\left(X_{\tau}\right)$ assuming that the holder choose to exercise the option at $\tau \in[0, T]$. Then, according to the theory of modern finance, see [17] for instance, the arbitrage free price, at time $t$, of the American option, assuming that the risk-free interest rate is zero, is given by the optimal stopping problem

$$
u(x, t)=\sup _{t \leq \tau \leq T} \mathbb{E}\left[\psi\left(X_{t}^{x, t}\right)\right]
$$

where the supremum is taken with respect to all stopping times $\tau$ with values in $[t, T]$. The main result in [20] states that if $u$ is the function in (1.4) then $\tilde{u}(x, t)=u(x, T-t)$ is, assuming certain restrictions on the obstacle $\psi$, a solution to a problem in the form (1.2), with $f \equiv 0, g \equiv \psi$ and $\Omega=\mathbb{R}^{N} \times[0, T]$, where in this case the operator $L$ is the Kolmogorov operator associated to $X$ :

$$
L=\frac{1}{2} \sum_{i, j=1}^{m}\left(\sigma \sigma^{T}\right)_{i j} \partial_{x_{i} x_{j}}+\sum_{i, j=1}^{N} b_{i j} x_{i} \partial_{x_{j}}-\partial_{t} .
$$


$\sigma^{T}$ denotes the transpose of $\sigma$. In the uniformly elliptic-parabolic case, $m=N$, the valuation of American options has bee quite thoroughly studied, see [2], [16] and [15]. However, there are significant classes of American options, commonly traded in financial markets, whose corresponding diffusion process $X$ is associated with Kolmogorov type operators which are not uniformly elliptic-parabolic, i.e., in particular $m<N$. Two such examples are provided by American Asian style options, see [1], and by American options priced in the stochastic volatility suggested in [13], see also [7] and [11]. Furthermore, as noted in [9] a general (mathematical) theory for American options in these settings is not yet available and the bulk of the literature focus mainly on numerical issues.

The purpose of this paper is to advance the mathematical theory for the obstacle problem for hypoelliptic ultraparabolic operators of Kolmogorov type and in particular to continue the study of the obstacle problem initiated in [9] and [20]. In [9], and the related work in [20], a number of important steps were taken towards developing a rigorous theory for the obstacle problem in (1.2) and the optimal stopping problem in (1.4). In particular, the main result in [9] is the existence, using the same set-up and assumption as in this paper, of a strong solution to the problem in (1.2) in certain bounded cylindrical domains and in the strip $\left.\mathbb{R}^{N} \times\right] 0, T$. Moreover, while the study in [9] was more directed towards existence results the main purpose of this paper is to prove optimal interior regularity for solutions to the problem (1.2).

To be able to proceed with our discussion and to properly state our results we next introduce the appropriate notation and describe the assumptions imposed on the operator $L$. Concerning structural assumptions on the operator $L$ and the problem in (1.2) we assume

H1 the coefficients $a_{i j}=a_{j i}$ are bounded continuous functions for $i, j=1, \ldots, m$. Moreover, there exists a positive constant $\lambda$ such that

$$
\lambda^{-1}|\xi|^{2} \leq \sum_{i, j=1}^{m} a_{i j}(x, t) \xi_{i} \xi_{j} \leq \lambda|\xi|^{2}, \quad \xi \in \mathbb{R}^{m},(x, t) \in \mathbb{R}^{N+1}
$$

H2 the operator

$$
K u:=\sum_{i=1}^{m} \partial_{x_{i} x_{i}} u+\sum_{i, j=1}^{N} b_{i j} x_{i} \partial_{x_{j}} u-\partial_{t} u
$$

is hypoelliptic, i.e. every distributional solution of $K u=f$ is a smooth solution, whenever $f$ is smooth;

H3 $a_{i j}, b_{i}$, for $i, j=1, \ldots, m$, and $f$ belong to the space $C_{K}^{0, \alpha}$ of Hölder continuous functions defined in (1.17), for some $\alpha \in] 0,1[$. The function $g$ in (1.2) is continuous in $\bar{\Omega}$.

Let

$$
Y=\sum_{i, j=1}^{N} b_{i j} x_{i} \partial_{x_{j}}-\partial_{t}
$$

and let $\operatorname{Lie}\left(Y, \partial_{x_{1}}, . ., \partial_{x_{m}}\right)$ denote the Lie algebra generated by the vector fields $Y, \partial_{x_{1}}, . ., \partial_{x_{m}}$. It is well-known that $\mathbf{H 2}$ can be stated in terms of the well-known Hörmander condition [14]:

$$
\operatorname{rank} \operatorname{Lie}\left(Y, \partial_{x_{1}}, . ., \partial_{x_{m}}\right)=N+1
$$


Yet another condition, equivalent to $\mathbf{H 2}$ and (1.7), is that there exists a basis for $\mathbb{R}^{N}$ such that the matrix $B$ has the form

$$
\left(\begin{array}{ccccc}
* & B_{1} & 0 & \cdots & 0 \\
* & * & B_{2} & \cdots & 0 \\
\vdots & \vdots & \vdots & \ddots & \vdots \\
* & * & * & \cdots & B_{\kappa} \\
* & * & * & \cdots & *
\end{array}\right)
$$

where $B_{j}$, for $j \in\{1, \ldots, \kappa\}$, is a $m_{j-1} \times m_{j}$ matrix of rank $m_{j}, 1 \leq m_{\kappa} \leq \ldots \leq m_{1} \leq m$ and $m+m_{1}+\ldots+m_{\kappa}=N$, while $*$ represents arbitrary matrices with constant entries. We also note that the natural setting for operators satisfying a Hörmander condition is that of the analysis on Lie groups. In particular, as shown in [18] the relevant Lie group related to the operator $K$ in (1.6) is defined using the group law

$$
(x, t) \circ(y, s)=(y+E(s) x, t+s), \quad E(s)=\exp \left(-s B^{T}\right), \quad(x, t),(y, s) \in \mathbb{R}^{N+1},
$$

where $B^{T}$ denotes the transpose of the matrix $B$. Moreover, if the matrices denoted by $*$ in (1.8) are null then there is, based on the block structure of $B$ defined in (1.8), a natural family of dilations

$$
D_{r}=\operatorname{diag}\left(r I_{m}, r^{3} I_{m_{1}}, . ., r^{2 \kappa+1} I_{m_{\kappa}}\right), \quad \delta_{r}=\operatorname{diag}\left(D_{r}, r^{2}\right), r>0,
$$

associated to the Lie group. In (1.14) $I_{k}, k \in \mathbb{N}$, is the $k$-dimensional unit matrix and $\delta_{r}$ is by definition a diagonal matrix. Moreover we set

$$
\mathbf{q}=m+3 m_{1}+\ldots+(2 \kappa+1) m_{\kappa},
$$

and we say that $\mathbf{q}+2$ is the homogeneous dimension of $\mathbb{R}^{N+1}$ defined with respect to the dilations $\left(\delta_{r}\right)_{r>0}$. Furthermore, we split the coordinate $x \in \mathbb{R}^{N}$ as $x=\left(x^{(0)}, x^{(1)}, \ldots, x^{(\kappa)}\right)$ where $x^{(0)} \in \mathbb{R}^{m}$ and $x^{(j)} \in \mathbb{R}^{m_{j}}$ for all $j \in\{1, . ., \kappa\}$. Based on this we define

$$
|x|_{K}=\sum_{j=0}^{\kappa}\left|x^{(j)}\right|^{\frac{1}{2 j+1}}, \quad\|(x, t)\|_{K}=|x|_{K}+|t|^{\frac{1}{2}}
$$

and we note that $\left\|\delta_{r}(x, t)\right\|_{K}=r\|(x, t)\|_{K}$ and we recall the following triangular inequality (cf. [10]): for any compact subset $H$ of $\mathbb{R}^{N+1}$, there exists a positive constant $c$ such that

$$
\left\|z^{-1}\right\|_{K} \leq c\|z\|_{K}, \quad\|z \circ w\|_{K} \leq c\left(\|z\|_{K}+\|w\|_{K}\right), \quad z, w \in H .
$$

We also define the quasi-distance $d_{K}$ by setting

$$
d_{K}(z, w):=\left\|w^{-1} \circ z\right\|_{K}, \quad w, z \in \mathbb{R}^{N+1} .
$$

Note that, for every compact set $H \subset \mathbb{R}^{N+1}$ we have

$$
d_{K}(z, w) \leq c d_{K}(w, z), \quad d_{K}(z, w) \leq c\left(d_{K}(z, \zeta)+d_{K}(\zeta, w)\right), \quad w, z, \zeta \in H .
$$

We finally set, for any $z \in \mathbb{R}^{N+1}$ and $H \subset \mathbb{R}^{N+1}$,

$$
d_{K}(z, H):=\inf \left\{d_{K}(z, w) \mid w \in H\right\} .
$$

To simplify our presentation we will also assume the following technical condition: 
$\mathbf{H} 4$ the operator $K$ is $\delta_{r}$-homogeneous of degree two with respect to the dilations group $\left(\delta_{r}\right)_{r>0}$ in $(1.10)$.

Note that, under assumption $\mathbf{H 4}$, the constant $c$ in (1.13) does not depend on $H$.

Concerning the regularity assumptions on the functions $a_{i j}, b_{i}, f, \psi$ and $g$ in (1.2) we will formulate these assumptions using certain anisotropic Hölder spaces defined based on $\|\cdot\|_{K}$. In particular, let $\alpha \in(0,1]$ and let $\Omega \subset \mathbb{R}^{N+1}$. We denote by $C_{K}^{0, \alpha}(\Omega), C_{K}^{1, \alpha}(\Omega)$ and $C_{K}^{2, \alpha}(\Omega)$ the Hölder spaces defined by the following norms:

$$
\begin{aligned}
& \|u\|_{C_{K}^{0, \alpha}(\Omega)}=\sup _{\Omega}|u|+\sup _{\substack{z, \zeta \in \Omega \\
z \neq \zeta}} \frac{|u(z)-u(\zeta)|}{\left\|\zeta^{-1} \circ z\right\|_{K}^{\alpha}}, \\
& \|u\|_{C_{K}^{1, \alpha}(\Omega)}=\|u\|_{C_{K}^{0, \alpha}(\Omega)}+\sum_{i=1}^{m}\left\|\partial_{x_{i}} u\right\|_{C_{K}^{0, \alpha}(\Omega)}+\sup _{\substack{z, \zeta \in \Omega \\
z \neq \zeta}} \frac{\left|u(z)-u(\zeta)-\sum_{j=1}^{m}\left(z_{j}-\zeta_{j}\right) \partial_{x_{j}} u(\zeta)\right|}{\left\|\zeta^{-1} \circ z\right\|_{K}^{1+\alpha}}, \\
& \|u\|_{C_{K}^{2, \alpha}(\Omega)}=\|u\|_{C_{K}^{0, \alpha}(\Omega)}+\sum_{i=1}^{m}\left\|\partial_{x_{i}} u\right\|_{C_{K}^{0, \alpha}(\Omega)}+\sum_{i, j=1}^{m}\left\|\partial_{x_{i} x_{j}} u\right\|_{C_{K}^{0, \alpha}(\Omega)}+\|Y u\|_{C_{K}^{0, \alpha}(\Omega)} .
\end{aligned}
$$

Moreover, we let $C^{0}(\Omega)$ denote the set of functions which are continuous on $\Omega$. Note that any $u \in C_{K}^{0, \alpha}(\Omega), \Omega$ bounded, is Hölder continuous in the usual sense since

$$
\left\|\zeta^{-1} \circ z\right\|_{K} \leq c_{\Omega} \mid z-\zeta^{\frac{1}{2 \kappa+1}}
$$

Remark 1.1 It is known (cf. for instance [6], Theorem 2.16, [19], Theorem 4 or [3]) that if $u \in C_{K}^{0, \alpha}(\Omega), \partial_{x_{j}} u \in C_{K}^{0, \alpha}(\Omega), j=1, \ldots, m$ and if

$$
|u(z \circ(0, s))-u(z)| \leq C|s|^{\frac{1+\alpha}{2}} \quad \text { whenever } z, z \circ(0, s) \in \Omega,
$$

then $u \in C_{K}^{1, \alpha}\left(\Omega^{\prime}\right)$ for every $\Omega^{\prime}$ compact subset of $\Omega$.

Let $k \in\{0,1,2\}, \alpha \in(0,1]$. If $\psi \in C_{K}^{k, \alpha}\left(\Omega^{\prime}\right)$ for every compact subset $\Omega^{\prime}$ of $\Omega$ then we write $\psi \in C_{K, \text { loc }}^{k, \alpha}(\Omega)$. Furthermore, for $p \in[1, \infty]$ we define the Sobolev-Stein spaces

$$
S^{p}(\Omega)=\left\{u \in L^{p}(\Omega): \partial_{x_{i}} u, \partial_{x_{i} x_{j}} u, Y u \in L^{p}(\Omega), i, j=1, \ldots, m\right\}
$$

and we let

$$
\|u\|_{S^{p}(\Omega)}=\|u\|_{L^{p}(\Omega)}+\sum_{i=1}^{m}\left\|\partial_{x_{i}} u\right\|_{L^{p}(\Omega)}+\sum_{i, j=1}^{m}\left\|\partial_{x_{i} x_{j}} u\right\|_{L^{p}(\Omega)}+\|Y u\|_{L^{p}(\Omega)} .
$$

If $u \in S^{p}(H)$ for every compact subset $H$ of $\Omega$ then we write $u \in S_{\text {loc }}^{p}(\Omega)$.

Definition 1.2 We say that $u \in \mathcal{S}_{\text {loc }}^{1}(\Omega) \cap C^{0}(\bar{\Omega})$ is a strong solution to problem (1.2) if the differential inequality is satisfied a.e. in $\Omega$ and the boundary datum is attained pointwise.

Under suitable assumptions, existence and uniqueness of a strong solution to (1.2) have been proved in [9] and [20]. 
To state our results we will make use of the following notation. For $x \in \mathbb{R}^{N}$ and $r>0$ we let $B_{r}(x)$ denote the open ball in $\mathbb{R}^{N}$ with center $x$ and radius $r$. We let $e_{1}$ be the unit vector pointing in the $x_{1}$-direction in the canonical base for $\mathbb{R}^{N}$. We let

$$
\begin{aligned}
Q & \left.=\left(B_{1}\left(\frac{1}{2} e_{1}\right) \cap B_{1}\left(-\frac{1}{2} e_{1}\right)\right) \times\right]-1,1[, \\
Q^{+} & =\left(B_{1}\left(\frac{1}{2} e_{1}\right) \cap B_{1}\left(-\frac{1}{2} e_{1}\right)\right) \times[0,1[, \\
Q^{-} & \left.\left.=\left(B_{1}\left(\frac{1}{2} e_{1}\right) \cap B_{1}\left(-\frac{1}{2} e_{1}\right)\right) \times\right]-1,0\right] .
\end{aligned}
$$

Then $Q$ is a space-time cylinder, $Q^{+}$will be referred to as the upper half-cylinder and $Q^{-}$will be referred to as the lower half-cylinder. We also let, whenever $(x, t) \in \mathbb{R}^{N+1}, r>0$,

$$
Q_{r}=\delta_{r}(Q), \quad Q_{r}(x, t)=(x, t) \circ Q_{r}, \quad Q_{r}^{ \pm}=\delta_{r}\left(Q^{ \pm}\right), \quad Q_{r}^{ \pm}(x, t)=(x, t) \circ Q_{r}^{ \pm} .
$$

Then $Q_{r}(x, t)$ is the cylinder $Q$ scaled to size $r$ and translated to the point $(x, t)$. We also note that the volume of $Q_{r}(x, t)$ is $r^{\mathbf{q}+2}$ times the volume of $Q$, where $\mathbf{q}$ is the homogeneous dimension in (1.11).

Remark 1.3 We set

$$
\tilde{d}_{K}((x, t),(\xi, \tau))=\inf \left\{r>0 \mid(x, t) \in Q_{r}(\xi, \tau)\right\}
$$

that defines a distance equivalent to $d_{K}$ in the sense that

$$
\tilde{c}^{-1} d_{K}((x, t),(\xi, \tau)) \leq \tilde{d}_{K}((x, t),(\xi, \tau)) \leq \tilde{c} d_{K}((x, t),(\xi, \tau)), \quad(x, t),(\xi, \tau) \in \mathbb{R}^{N+1}
$$

for some positive constant $\tilde{c}$. It turns out that $Q_{r}(\xi, \tau)$ is the ball of radius $r$ and centered at $(\xi, \tau)$ with respect to the distance $\tilde{d}_{K}$. By (1.15), for any $r_{0}>0$ there exists a positive constant c such that:

i) if $(x, t) \in Q_{r}(\xi, \tau)$ then $(\xi, \tau) \in Q_{c r}(x, t)$ for $\left.r \in\right] 0, r_{0}[$;

ii) if $(x, t) \in Q_{r}(\xi, \tau)$ then $Q_{\rho}(x, t) \in Q_{c(r+\rho)}(\xi, \tau)$ for $\left.r, \rho \in\right] 0, r_{0}[$.

We also note that as a consequence we have that if $(x, t) \in Q_{r}(\xi, \tau)$ then

$$
\left.Q_{r}(\xi, \tau) \subseteq Q_{C_{1} r}(x, t) \quad r \in\right] 0, r_{0}[
$$

for some positive constant $C_{1}$.

The main reason we work with these cylinders is that these cylinders are regular for the Dirichlet problem for the operators considered in this paper. In particular, the following theorem holds.

Theorem 1.4 (TheOREM 4.2 IN [10]) Assume hypotheses H1-3. For any $R>0$ and $(x, t) \in$ $\mathbb{R}^{N+1}$, there exists a unique classical solution $u \in C_{K, l o c}^{2, \alpha}\left(Q_{R}(x, t)\right) \cap C^{0}\left(Q_{R}(x, t) \cup \partial_{P} Q_{R}(x, t)\right)$ to the Dirichlet problem

$$
\begin{cases}L u=f, & \text { in } Q_{R}(x, t), \\ u=g, & \text { on } \partial_{P} Q_{R}(x, t) .\end{cases}
$$


We can now state the three main theorems proved in this paper. In the following, we use the notation

$$
\mathbf{c}_{\alpha}=\sum_{i, j=1}^{m}\left\|a_{i j}\right\|_{C_{K}^{0, \alpha}(\Omega)}+\sum_{j=1}^{m}\left\|b_{j}\right\|_{C_{K}^{0, \alpha}(\Omega)} .
$$

Theorem 1.5 Assume hypotheses H1-4 with $\Omega=Q$. Let $\psi \in C_{K}^{0, \alpha}(Q)$ be such that $\psi \leq g$ on $\partial_{P} Q$. If $u$ is a strong solution to problem (1.2) in $Q$, then $u \in C_{K}^{0, \alpha}\left(Q_{R}\right)$ and

$$
\|u\|_{C_{K}^{0, \alpha}\left(Q_{R}\right)} \leq c\left(N, \lambda, \alpha, \mathbf{c}_{\alpha},\|f\|_{C_{K}^{0, \alpha}(Q)},\|g\|_{L^{\infty}(Q)},\|\psi\|_{C_{K}^{0, \alpha}(Q)}\right)
$$

for some $R \in] 0,1[$.

Theorem 1.6 Assume hypotheses H1-4 with $\Omega=Q$. Let $\psi \in C_{K}^{1, \alpha}(Q)$ be such that $\psi \leq g$ on $\partial_{P} Q$. If $u$ is a strong solution to problem (1.2) in $Q$, then $u \in C_{K}^{1, \alpha}\left(Q_{R}\right)$ and

$$
\|u\|_{C_{K}^{1, \alpha}\left(Q_{R}\right)} \leq c\left(N, \lambda, \alpha, \mathbf{c}_{\alpha},\|f\|_{C_{K}^{0, \alpha}(Q)},\|g\|_{L^{\infty}(Q)},\|\psi\|_{C_{K}^{1, \alpha}(Q)}\right)
$$

for some $R \in] 0,1[$.

Theorem 1.7 Assume hypotheses H1-4 with $\Omega=Q$. Let $\psi \in C_{K}^{2, \alpha}(Q)$ be such that $\psi \leq g$ on $\partial_{P} Q$. If $u$ is a strong solution to problem (1.2) in $Q$, then $u \in S^{\infty}\left(Q_{R}\right)$ and

$$
\|u\|_{S^{\infty}\left(Q_{R}\right)} \leq c\left(N, \lambda, \alpha, \mathbf{c}_{\alpha},\|f\|_{C_{K}^{0, \alpha}(Q)},\|g\|_{L^{\infty}(Q)},\|\psi\|_{C_{K}^{2, \alpha}(Q)}\right)
$$

for some $R \in] 0,1[$.

We note that Theorem 1.5, Theorem 1.6 and Theorem 1.7 concern the optimal interior regularity for the solution $u$ to the obstacle problem under different assumption on the regularity of the obstacle $\psi$. In particular, the main differences between the theorems concern the smoothness of the obstacle and Theorem 1.5 and Theorem 1.6 treat the case of non-smooth obstacles while Theorem 1.7 treats the case of smooth obstacles. Whether or not an obstacle is smooth or not is simply determined by whether or not $L \psi$ is pointwise well-defined. Moreover, the results stated in the theorems are the same: the solution is, up to $S^{\infty}$-smoothness, as smooth as the obstacle.

Concerning previous results we note that in the uniformly elliptic-parabolic case, $m=N$, and in the case of smooth obstacles, there is a literature on the existence and regularity of solutions to the problem in (1.2) and we refer to [12], 22], [23] and [4]. However, in the case of non-smooth obstacles not very much is known, for the general problem in (1.2), even in the uniformly ellipticparabolic case, $m=N$, as there in this case are not many techniques available for the study of the obstacle problem. However, in a recent paper [21] the theory for the obstacle problem in the uniformly elliptic-parabolic case, $m=N$, was advanced also in the case of non-smooth obstacle. Moreover, the approach outlined in [21] applies as well to certain fully non-linear parabolic equations, see [21].

Concerning previous results in the non-uniformly elliptic-parabolic case, $m<N$, there are to our knowledge no results available in the literature, even in the case of smooth obstacles, of the type presented in this paper. In particular, our main theorems, Theorems 1.5, 1.6 and 1.7, are completely new. Concerning proofs, our arguments use blow-up techniques similar to 
21] combined with several results concerning equations of Kolmogorov type established by the third and fourth author with collaborators. We refer to the bulk of the paper for details of the arguments. Finally we note that in future papers we intend to study the underlying free boundary with the ambition to develop a regularity theory for free boundaries in the setting of hypoelliptic ultraparabolic operator of Kolmogorov type.

The rest of this paper is organized as follows. In section 2 we collect a number of important facts concerning operators of Kolmogorov type. In section 3 we then prove our main results, i.e., Theorems 1.5, 1.6 and 1.7.

\section{Preliminaries on operators of Kolmogorov type}

In this section we collect a number of results concerning operators of Kolmogorov type to be used in the proof of Theorems 1.5, 1.6 and 1.7.

Theorem 2.1 (TheOREM 1.3 IN [10]) Assume hypotheses H1-3. Let $R>0$ and $(x, t) \in \mathbb{R}^{N+1}$. If $u \in C_{K, l o c}^{2, \alpha}\left(Q_{R}(x, t)\right)$ satisfies $L u=f$ in $Q_{R}(x, t)$, then there exists a positive constant $c$, depending on $N, \alpha, \mathbf{c}_{\alpha}, \lambda$ and $R$, such that

$$
\|u\|_{C_{K}^{2, \alpha}\left(Q_{R / 2}(x, t)\right)} \leq c\left(\|u\|_{L^{\infty}\left(Q_{R}(x, t)\right)}+\|f\|_{C_{K}^{0, \alpha}\left(Q_{R}(x, t)\right)}\right) .
$$

Theorem 2.2 (THEOREM 1.4 IN [8]) Assume hypotheses H1-3. There exists a fundamental solution $\Gamma$ to the operator $L$ in (1.1). More precisely, a classical solution to the Cauchy problem

$$
\begin{cases}L u=f, & \text { in } \left.\mathbb{R}^{N} \times\right] 0, T[ \\ u=g, & \text { in } \mathbb{R}^{N}\end{cases}
$$

is given by

$$
u(x, t)=\int_{\mathbb{R}^{N}} \Gamma(x, t, y, 0) g(y) d y+\int_{0}^{t} \int_{\mathbb{R}^{N}} \Gamma(x, t, y, s) f(y, s) d y d s,
$$

whenever $f \in C_{K, \text { loc }}^{0, \alpha}\left(\mathbb{R}^{N} \times\right] 0, T[)$ and $g \in C^{0}\left(\mathbb{R}^{N}\right)$ are bounded functions. Formula (2.2) also holds whenever $f$ and $g$ satisfy the following growth conditions: there exists a positive $M$ such that

$$
\left.|f(x, t)| \leq M e^{M|x|^{2}}, \quad|g(x)| \leq M e^{M|x|^{2}}, \quad(x, t) \in \mathbb{R}^{N} \times\right] 0, T[.
$$

In this case $T$ has to be sufficiently small (depending on $M$ ). Furthermore, $u$ in (2.2) is the unique solution to the problem in (2.1) in the class of all functions satisfying (2.3).

Let $\Gamma^{\mu}$ denote the fundamental solution to the constant coefficient Kolmogorov operator

$$
K^{\mu}=\mu \sum_{i=1}^{m} \partial_{x_{i} x_{i}}+\sum_{i, j=1}^{N} b_{i j} x_{i} \partial_{x_{j}}-\partial_{t}
$$

for $\mu>0$. Combining [8], Theorem 1.4, and [10], Theorem 1.5, we have the following theorem. 
Theorem 2.3 Under hypotheses H1-3, there exist four positive constants $\mu^{-}, \mu^{+}, c^{-}, c^{+}$such that

$$
c^{-} \Gamma^{\mu^{-}}(x, t, y, s) \leq \Gamma(x, t, y, s) \leq c^{+} \Gamma^{\mu^{+}}(x, t, y, s)
$$

for every $(x, t),(y, s) \in \mathbb{R}^{N+1}$, and $0<t-s<T$. We have $\mu^{-}<\lambda<\mu^{+}$, where $\lambda$ is the constant in $\mathbf{H 1}, \mu^{+}$can be chosen arbitrarily close to $\lambda$ and $c^{+}$and $c^{-}$depend on $\mu^{+}$and on $T$.

We note that the fundamental solution $\Gamma^{\mu}$ can be given explicitly. Let

$$
\mathcal{C}(t):=\int_{0}^{t} E(s)\left(\begin{array}{cc}
I_{m} & 0 \\
0 & 0
\end{array}\right) E^{T}(s) d s, \quad t \in \mathbb{R}^{N},
$$

where the matrix $I_{m}$ equals the $m \times m$-identity matrix and $E(s)$ is defined as in (1.9). It is well known, see e.g. [18], that $\mathbf{H 2}$ and (1.7) are equivalent to the condition that

$$
\mathcal{C}(t)>0 \text { for all } t>0 .
$$

Assuming that (2.5) holds, we have that

$$
\Gamma^{\mu}(x, t, y, s)=\Gamma^{\mu}(x-E(t-s) y, t-s, 0,0)
$$

where $\Gamma^{\mu}(x, t, 0,0)=0$ if $t \leq 0$ and

$$
\Gamma^{\mu}(x, t, 0,0)=\frac{(4 \pi \mu)^{-N / 2}}{\sqrt{\operatorname{det} \mathcal{C}(t)}} \exp \left(-\frac{1}{4 \mu}\left\langle\mathcal{C}(t)^{-1} x, x\right\rangle-t \operatorname{Tr}(B)\right) \quad \text { if } t>0 .
$$

We also note that

$$
\left.\Gamma^{\mu}(x, t, y, s) \leq \frac{c(T)}{\left\|(y, s)^{-1} \circ(x, t)\right\|_{K}^{\mathbf{q}}} \text { for all }(x, t),(y, s) \in \mathbb{R}^{N} \times\right] 0, T[, t>s,
$$

where $\mathbf{q}$ was introduced in (1.11). For (2.8) we refer to [10], Proposition 2.8.

Assumption $\mathbf{H 4}$ implies that the following identities hold:

$$
\mathcal{C}\left(r^{2} t\right)=D_{r} \mathcal{C}(t) D_{r}, \quad E\left(r^{2} t\right) D_{r}=D_{r} E(t), \quad t \in \mathbb{R}, r>0,
$$

so that in particular we have

$$
\Gamma^{\mu}(x, t, 0,0)=\frac{(4 \pi \mu)^{-N / 2}}{\sqrt{t^{\mathbf{q}} \operatorname{det} \mathcal{C}(1)}} \exp \left(-\frac{1}{4 \mu}\left\langle\mathcal{C}(1)^{-1} D_{\frac{1}{\sqrt{t}}} x, D_{\frac{1}{\sqrt{t}}} x\right\rangle\right) \quad \text { if } t>0 .
$$

Some analogous formulas also hold in general. Specifically, for every positive $T$ there exist two positive constants $c_{T}^{\prime}$ and $c_{T}^{\prime \prime}$ such that

$$
\begin{array}{r}
c_{T}^{\prime}\left\langle\mathcal{C}^{-1}(1)\left(D_{\frac{1}{\sqrt{t}}} x+E(1) D_{\frac{1}{\sqrt{t}}} y\right), D_{\frac{1}{\sqrt{t}}} x+E(1) D_{\frac{1}{\sqrt{t}}} y\right\rangle \leq \\
\left\langle\mathcal{C}^{-1}(t)(x+E(t) y), x+E(t) y\right\rangle \leq \\
c_{T}^{\prime \prime}\left\langle\mathcal{C}^{-1}(1)\left(D_{\frac{1}{\sqrt{t}}} x+E(1) D_{\frac{1}{\sqrt{t}}} y\right), D_{\frac{1}{\sqrt{t}}} x+E(1) D_{\frac{1}{\sqrt{t}}} y\right\rangle,
\end{array}
$$

for every $\left.\left.(x, t) \in \mathbb{R}^{N} \times\right] 0, T\right]$ (see (2.16), and (2.18) in [5]), and, as a plain consequence,

$$
\left.\left.\tilde{c}_{T}^{\prime} t^{\mathbf{q}} \leq \operatorname{det} \mathcal{C}(t) \leq \tilde{c}_{T}^{\prime \prime} t^{\mathbf{q}}, \quad t \in\right] 0, T\right]
$$

In the forthcoming sections we will need the following technical estimate. 
Corollary 2.4 Under assumptions H1-3, we define, for $\gamma>0$ and $R_{0}>1$, the function

$$
u(x, t)=\int_{|y|_{K} \leq R_{0}} \Gamma(x, t, y, 0)|y|_{K}^{\gamma} d y, \quad x \in \mathbb{R}^{N}, t>0 .
$$

For every compact subset $H \subset \mathbb{R}^{N+1}$, there exists a positive constant $c=c\left(\gamma, R_{0}, H\right)$ such that

$$
u(x, t) \leq c\|(x, t)\|_{K}^{\gamma}, \quad(x, t) \in H .
$$

Proof. By the triangle inequalities (1.13), we have

$$
|y|_{K}=\|(y, 0)\|_{K} \leq c\left\|(y, 0)^{-1}\right\|_{K} \leq c^{2}\left(\left\|(y, 0)^{-1} \circ(x, t)\right\|_{K}+c\|(x, t)\|_{K}\right),
$$

for any $x, y \in \mathbb{R}^{N}$ and $t \in \mathbb{R}$. By Theorem 2.3 , we have

$$
\begin{aligned}
u(x, t) & \leq c_{+} \int_{|y|_{K} \leq R_{0}} \Gamma^{+}(x, t, y, 0)|y|_{K}^{\gamma} d y \leq c^{\prime}\|(x, t)\|_{K}^{\gamma} \int_{|y|_{K} \leq R_{0}} \Gamma^{+}(x, t, y, 0) d y \\
& +c^{\prime \prime} \int_{|y|_{K} \leq R_{0}} \Gamma^{+}\left((y, 0)^{-1} \circ(x, t)\right)\left\|(y, 0)^{-1} \circ(x, t)\right\|_{K}^{\gamma} d y .
\end{aligned}
$$

We perform the change of variables

$$
w=\delta_{\frac{1}{\sqrt{t}}}\left((y, 0)^{-1} \circ(x, t)\right)=(\xi, 1), \quad \xi=D_{\frac{1}{\sqrt{t}}}(x-E(-t) y),
$$

and, by (2.11) and (2.12), we obtain

$$
u(x, t) \leq c^{\prime}\|(x, t)\|_{K}^{\gamma}+c^{\prime \prime} t^{\frac{\gamma}{2}} .
$$

Obviously this estimate completes the proof of the lemma.

We end this section by proving two further results useful in the proof of Theorems 1.5-1.7. The first one is a version of the Harnack inequality for non-negative solution $u$ of $L u=0$ proved in [10] and the second one is a version of an estimate in "thin cylinders" proved in [5]. We first need to introduce some notations. For any positive $T, R$, and $\left(x_{0}, t_{0}\right) \in \mathbb{R}^{N+1}$ we put $Q^{-}(T)=\left(B_{1}\left(\frac{1}{2} e_{1}\right) \cap B_{1}\left(-\frac{1}{2} e_{1}\right)\right) \times[-T, 0]$, and $Q_{R}^{-}\left(x_{0}, t_{0}, T\right)=\left(x_{0}, t_{0}\right) \circ \delta_{R}\left(Q^{-}\left(R^{-2} T\right)\right)$. Note that, from (1.10) it follows that $T$ is the true height of $Q_{R}^{-}\left(x_{0}, t_{0}, T\right)$. For $\alpha, \beta, \gamma \in \mathbb{R}$, with $0<\alpha<\beta<\gamma<1$, we set

$$
\begin{aligned}
& \widetilde{Q}_{R}^{-}\left(x_{0}, t_{0}, T\right)=\left\{(x, t) \in Q_{R}^{-}\left(x_{0}, t_{0}, T\right) \mid t_{0}-\gamma T \leq t \leq t_{0}-\beta T\right\}, \\
& \widetilde{Q}_{R}^{+}\left(x_{0}, t_{0}, T\right)=\left\{(x, t) \in Q_{R}^{-}\left(x_{0}, t_{0}, T\right) \mid t_{0}-\alpha T \leq t \leq t_{0}\right\} .
\end{aligned}
$$

We recall the following invariant Harnack inequality for non-negative solutions $u$ of $L u=0$.

Theorem 2.5 (THEOREM 1.2 IN [10]) Under assumptions $\mathbf{H 1 - 3}$, there exist constants $R_{0}>0$, $M>1$ and $\alpha, \beta, \gamma, \varepsilon \in] 0,1[$, with $0<\alpha<\beta<\gamma<1$, depending only on the operator $L$, such that

$$
\sup _{\widetilde{Q}_{\varepsilon R}^{-}\left(x_{0}, t_{0}, R^{2}\right)} u \leq M \inf _{\widetilde{Q}_{\varepsilon R}^{+}\left(x_{0}, t_{0}, R^{2}\right)} u
$$

for every positive solution $u$ of $L u=0$ in $Q_{R}^{-}\left(x_{0}, t_{0}\right)$ and for any $\left.R \in\right] 0, R_{0}\left[,\left(x_{0}, t_{0}\right) \in \mathbb{R}^{N+1}\right.$. 
Our first preliminary result is the following

Lemma 2.6 Assume H1-3. For any $T>0$ and $\tilde{R} \geq \sqrt{2 T+1}$ there exist constants $c=c\left(\alpha, \mathbf{c}_{\alpha}\right)$ and $\tilde{c}=c\left(\alpha, \mathbf{c}_{\alpha}, \kappa, \tilde{R}\right)$ such that

$$
\sup _{Q_{\tilde{R}}^{-} \cap\{(x, t): t=-2 T\}} u \leq \tilde{c} \inf _{Q_{\tilde{R} / 2}^{-}(0,0, T)} u
$$

for any positive solution $u$ to $L u=0$ in $Q_{\bar{R}}^{-}(0,0,2 T+1)$ with $\bar{R} \geq c \tilde{R}^{2 \kappa+1}$.

Proof. Let $u: Q_{\bar{R}}^{-}(0,0,2 T+1) \longrightarrow \mathbb{R}$ be a positive solution of $L u=0$, where $\bar{R}$ is a suitably large constant that will be chosen lather. We aim to show that, for every $(x, t) \in Q_{\tilde{R} / 2}^{-}(0,0, T)$, and $(y,-2 T) \in Q_{\tilde{R}}^{-}$, there exists a Harnack chain connecting $(x, t)$ to $(y,-2 T)$. Specifically, we prove the existence of a finite sequence $\left(R_{j}\right)_{j=1, \ldots, k}$ such that $0<R_{j} \leq R_{0}$, for any $j=1, \ldots, k$ and a sequence of points $\left(x_{j}, t_{j}\right)_{j=1, \ldots, k}$ such that $\left(x_{1}, t_{1}\right)=(x, t)$,

$$
Q_{R_{j}}^{-}\left(x_{j}, t_{j}\right) \subset Q_{\bar{R}}^{-}(0,0,2 T+1),
$$

with $\left(x_{j+1}, t_{j+1}\right) \in \widetilde{Q}_{\varepsilon R_{j}}^{-}\left(x_{j}, t_{j}, R_{j}^{2}\right)$, for every $j=1, \ldots, k-1$ and $(y,-2 T) \in \widetilde{Q}_{\varepsilon R_{k}}^{-}\left(x_{k}, t_{k}, R_{k}^{2}\right)$. Using this construction and Theorem 2.5 we then find that $u\left(x_{j}, t_{j}\right) \leq M u\left(x_{j-1}, t_{j-1}\right), j=$ $1, \ldots, k$, and that

$$
u(y,-2 T) \leq M u\left(x_{k}, t_{k}\right) \leq M^{k} u(x, t) .
$$

To prove the existence of a Harnack chain connecting $(x, t)$ to $(y,-2 T)$ as above, we apply the method previously used in the proof of Theorem 1.5 of [8]. The method concerns the problem of finding the shortest Harnack chain, in order to minimize the integer $k$. It turns out that the best choice is $\left(x_{j}, t_{j}\right)=\left(\gamma\left(\tau_{j}\right), t-\tau_{j}\right)$, where

$$
\gamma(\tau)=E(-\tau)\left(x+\mathcal{C}(\tau) \mathcal{C}^{-1}(t+2 T)(E(t+2 T) y-x)\right)
$$

and $\tau_{1}, \ldots, \tau_{k}$ are suitable real numbers such that $\tau_{1}=0<\tau_{2}<\cdots<\tau_{k}<t-2 T$. We finally have

$$
k \leq 1+\frac{1}{h}\left\langle\mathcal{C}^{-1}(t+2 T)(x-E(t+2 T) y), x-E(t+2 T) y\right\rangle
$$

for some positive constant $h$ only depending on the operator $L$ (we refer to [8] for more details). Since the function in (2.15) continuously depends on $(x, t)$ and $(y,-2 T)$, the inequality stated in Theorem 2.5 holds with

$$
\tilde{c}:=\max \left\{M^{1+\frac{1}{h}\left\langle\mathcal{C}^{-1}(t+2 T)(x-E(t+2 T) y), x-E(t+2 T) y\right\rangle} \mid(x, t, y,-2 T) \in \overline{Q_{\tilde{R} / 2}^{-}(0,0, T) \times Q_{\tilde{R}}^{-}}\right\},
$$

provided that $(2.13)$ holds for $j=1, \ldots, k$.

To conclude the proof of Lemma 2.6, it is sufficient to show that (2.13) holds for $j=1, \ldots, k$, as soon as $R$ is suitably large. In fact, we will prove that

$$
Q^{-}(\gamma(\tau), t-\tau) \subset Q_{\bar{R}}^{-}(0,0,2 T+1) \quad \text { for every } \tau \in[0, t+2 T],
$$

holds (recall that $t \in[-T, 0]$ ) and we note that this is stronger statement compared to (2.13).

To proceed we first note that $\left.\left.Q^{-}(\gamma(\tau), t-\tau) \subset \mathbb{R}^{N} \times\right]-2 T-1,0\right]$ for every $\tau \in[0, t+2 T]$. Concerning the lateral boundary of $Q_{\bar{R}}^{-}$, we consider any $(x, t) \in \overline{Q_{\tilde{R} / 2}^{-}(0,0, T)}$ and $(y,-2 T) \in \overline{Q_{\tilde{R}}^{-}}$. 
We have that $\left|x_{j}\right| \leq(\tilde{R} / 2)^{2 \kappa+1}$ and $\left|y_{j}\right| \leq \tilde{R}^{2 \kappa+1}$, for $j=, \ldots, N$. Then, by the continuity of $\gamma$ in (2.14), there exists a positive constant $c_{0}$ such that $|\gamma(\tau)| \leq c_{0} \tilde{R}^{2 \kappa+1}$, for every $\tau \in[0, t+2 T]$. Consider now any point $\left(\xi_{0}, \tau_{0}\right) \in Q^{-}(\gamma(\tau), t-\tau)$. There exists $\left(\xi_{1}, \tau_{1}\right) \in Q^{-}$such that

$$
\left(\xi_{0}, \tau_{0}\right)=(\gamma(\tau), t-\tau) \circ\left(\xi_{1}, \tau_{1}\right)=\left(\xi_{1}+E\left(\tau_{1}\right) \gamma(\tau), t-\tau+\tau_{1}\right) .
$$

As a consequence, there exists a positive constant $C_{1}$ such that

$$
\left|\xi_{0}\right| \leq\left|\xi_{1}\right|+\left|E\left(\tau_{1}\right) \gamma(\tau)\right| \leq C_{1}(1+|\gamma(\tau)|) \leq C_{1}\left(1+c_{0} \tilde{R}^{2 \kappa+1}\right)
$$

Hence, if we set $c=2 \kappa c_{0} C_{1}$, and we choose $\bar{R} \geq c \tilde{R}^{2 \kappa+1}$, we have $\left(\xi_{0}, \tau_{0}\right) \in Q_{\bar{R}}^{-}$for every $\left(\xi_{0}, \tau_{0}\right) \in Q^{-}(\gamma(\tau), t-\tau)$. This proves (2.16) and hence the proof of Lemma 2.6 is complete.

Lemma 2.7 Assume H1-3. Let $R>0$ be given. Then there exist constants $R_{0}, C_{0}, C_{1}>0$, $R_{0} \geq 2 R$, such that

$$
\sup _{Q_{R}^{-}}|v| \leq C_{0} e^{-C_{1} \tilde{R}^{2}} \sup _{\partial_{P} Q_{\tilde{R}}^{-} \cap\left\{(x, t): t>-R^{2}\right\}}|v|
$$

for any $\tilde{R} \geq R_{0}$ and for every $v$ solution of $L v=0$ in $Q_{\tilde{R}}^{-}\left(0,0, R^{2}\right)$ such that $v\left(\cdot,-R^{2}\right)=0$.

Proof. To prove this lemma we proceed as in the proof of Theorem 3.1 of [5]. We let $\tilde{R}$ be suitably large and to be chosen. Let $r>0$ be such that $\left\{|y|_{K} \leq 2 r\right\} \subset B_{1}\left(\frac{1}{2} e_{1}\right) \cap B_{1}\left(-\frac{1}{2} e_{1}\right)$ and let $\varphi \in C^{\infty}\left(\mathbb{R}^{N}\right)$ be a non-negative function such that $\varphi(x)=1$ if $|x|_{K} \geq 2 r$, and $\varphi(x)=0$ if $|x|_{K} \leq r$. We define

$$
w(x, t):=\frac{2}{c^{-}} \int_{\mathbb{R}^{N}} \Gamma\left(x, t, y,-R^{2}\right) \varphi\left(D_{1 / \tilde{R}} y\right) d y,
$$

where $c^{-}$the constant in Theorem 2.3 , related to $T=R^{2}$. Clearly, $w$ is a non-negative solution to the Cauchy problem $L u=0$ in $\left.\left.\mathbb{R}^{N} \times\right]-R^{2}, 0\right], u\left(x,-R^{2}\right)=\varphi\left(D_{1 / \tilde{R}^{x}}\right)$.

We note that, if $(x, t) \in \partial_{P} Q_{\tilde{R}}^{-}$is such that $t>-R^{2}$, then $\delta_{1 / \tilde{R}}(x, t) \in \partial_{P} Q^{-}\left(0,0, R^{2} / \tilde{R}^{2}\right)$. Moreover, for such $(x, t)$ we deduce using Theorem 2.3 that

$$
w(x, t) \geq 2 \int_{\mathbb{R}^{N}} \Gamma^{\mu^{-}}\left(x, t, y,-R^{2}\right) \varphi\left(D_{1 / \tilde{R}} y\right) d y .
$$

We next show that

$$
\int_{\mathbb{R}^{N}} \Gamma^{\mu^{-}}\left(x, t, y,-R^{2}\right) \varphi\left(D_{1 / \tilde{R}} y\right) d y \rightarrow 1, \quad \text { as } \quad \tilde{R} \rightarrow+\infty,
$$

uniformly uniformly in $(x, t) \in \partial_{P} Q_{\tilde{R}}^{-}$. Thus, there exists a positive $R_{0}$ such that, if $\tilde{R}>R_{0}$, we have $w(x, t) \geq 1$ for every $(x, t) \in \partial_{P} Q_{\tilde{R}}^{-} \cap\left\{(x, t): t>-R^{2}\right\}$. Thus, by our assumption on $v$ we see that the maximum principle implies that

$$
v(x, t) \leq w(x, t) \sup _{\partial_{P} Q_{\tilde{R}}^{-} \cap\left\{t>-R^{2}\right\}}|v| .
$$


We next prove (2.17). By (2.11) and (2.12) we have that $\Gamma^{\mu^{-}}(x, t, y, s) \leq \frac{c_{T}^{-}}{(t-s)^{\frac{\mathbf{q}}{2}}} \exp \left(-C_{T}^{-}\left\langle\mathcal{C}^{-1}(1)\left(D_{\frac{1}{\sqrt{t-s}}} x-E(1) D_{\frac{1}{\sqrt{t-s}}} y\right), D_{\frac{1}{\sqrt{t-s}}} x-E(1) D_{\frac{1}{\sqrt{t-s}}} y\right\rangle\right)$ for every $(x, t),(y, s) \in \mathbb{R}^{N+1}$ such that $0<t-s<T$. Then

$$
\begin{aligned}
& \left|\int_{\mathbb{R}^{N}} \Gamma^{\mu^{-}}\left(x, t, y,-R^{2}\right) \varphi\left(D_{1 / \tilde{R}} y\right) d y-1\right| \leq \\
& c_{T}^{-}\left(t+R^{2}\right)^{-\frac{\mathbf{q}}{2}} \int_{\mathbb{R}^{N}} \exp \left(-C_{T}^{-}\left\langle\mathcal{C}^{-1}(1)\left(D_{\frac{1}{\sqrt{t+R^{2}}}} x-E(1) D_{\frac{1}{\sqrt{t+R^{2}}}} y\right),\right.\right. \\
& \left.\left.D_{\frac{1}{\sqrt{t+R^{2}}}} x-E(1) D_{\frac{1}{\sqrt{t+R^{2}}}} y\right\rangle\right)\left|\varphi\left(D_{1 / \tilde{R}} y\right)-1\right| d \eta \leq \\
& c_{T}^{-}\left(\frac{\tilde{R}^{2}}{t+R^{2}}\right)^{\frac{\mathrm{q}}{2}} \int_{\mathbb{R}^{N}} \exp \left(-C_{T}^{-}\left\langle\mathcal{C}^{-1}(1)\left(D_{\frac{1}{\sqrt{t+R^{2}}}} x-E(1) D_{\frac{\tilde{R}}{\sqrt{t+R^{2}}}} \eta\right),\right.\right. \\
& \left.\left.D_{\frac{1}{\sqrt{t+R^{2}}}} x-E(1) D_{\frac{\tilde{R}}{\sqrt{t+R^{2}}}} \eta\right\rangle\right)|\varphi(\eta)-1| d \eta
\end{aligned}
$$

A direct computation shows that

$$
\tau^{-\frac{\mathbf{q}}{2}} \int_{\mathbb{R}^{N}} \exp \left(-C_{T}^{-}\left\langle\mathcal{C}^{-1}(1)\left(\xi-E(1) D_{\frac{1}{\sqrt{\tau}}} \eta\right), \xi-E(1) D_{\frac{1}{\sqrt{\tau}}} \eta\right\rangle\right)|\varphi(\eta)-1| d \eta \longrightarrow 0
$$

as $\tau \rightarrow 0^{+}$, uniformly for $2 r \leq|\xi|_{K} \leq 1$. This concludes the proof of (2.17).

To complete the proof of the lemma we see that it is enough to prove an upper bound for $w$ in the set $Q_{R}^{-}$. To do this we note that by Theorem 2.3, by (2.11), (2.11) 2 and the definition of $\varphi$, we have

$$
\begin{gathered}
w(x, t) \leq 2 \frac{c^{+}}{c^{-}} \int_{\mathbb{R}^{N}} \Gamma^{\mu^{+}}\left(x, t, y,-R^{2}\right) \varphi\left(D_{1 / \tilde{R}} y\right) d y \leq 2 \frac{c^{+}}{c^{-}} \int_{|y|_{K} \geq r \tilde{R}} \Gamma^{\mu^{+}}\left(x, t, y,-R^{2}\right) d y \\
\leq \frac{c_{T}^{+}}{\left(t+R^{2}\right)^{\mathbf{q} / 2}} \int_{|y|_{K} \geq r \tilde{R}} \exp \left(-C_{T}^{+}\left\langle\mathcal{C}^{-1}(1)\left(D_{\frac{1}{\sqrt{t+R^{2}}}} x-E(1) D_{\frac{1}{\sqrt{t+R^{2}}}} y\right),\right.\right. \\
\left.\left.D_{\frac{1}{\sqrt{t+R^{2}}}} x-E(1) D_{\frac{1}{\sqrt{t+R^{2}}}} y\right\rangle\right) d y .
\end{gathered}
$$

If we set $\mathcal{Q}:=E(1)^{T} \mathcal{C}^{-1}(1) E(1)$, we have that $\mathcal{Q}$ is a symmetric strictly positive constant matrix. Then, by the change of variable $\eta=D_{\frac{1}{\sqrt{t+R^{2}}}} y$ in (2.19), we get

$$
w(x, t) \leq c_{0} \int_{|\eta|_{K} \geq \frac{r \widetilde{R}}{\sqrt{t+R^{2}}}} \exp \left(-c^{+}\left\langle\mathcal{Q}\left(\eta-E(-1) D_{\frac{1}{\sqrt{t+R^{2}}}} x\right), \eta-E(-1) D_{\frac{1}{\sqrt{t+R^{2}}}} x\right\rangle\right) d \eta
$$

We next note that, since $(x, t) \in Q_{R}^{-}$, we have $t \in\left[-R^{2}, 0\right]$ and hence the norm $\left|E(-1) D_{\frac{1}{\sqrt{t+R^{2}}}} x\right|$ is bounded by a constant. On the other hand,

$$
\langle\mathcal{Q} \eta, \eta\rangle \geq \lambda_{\mathcal{Q}}\langle\eta, \eta\rangle=\lambda_{\mathcal{Q}} \sum_{j=0}^{\kappa} \frac{\left|\eta^{(j)}\right|^{2}}{|\eta|_{K}^{4 j+2}}|\eta|_{K}^{4 j+2} \geq \lambda_{\mathcal{Q}}|\eta|_{K}^{2} \sum_{j=0}^{\kappa} \frac{\left|\eta^{(j)}\right|^{2}}{|\eta|_{K}^{4 j+2}}
$$


since $|\eta|_{K} \geq \frac{r \tilde{R}}{\sqrt{t+R^{2}}}>1$ (for $R_{0}$ suitably large). As a consequence, there exists a positive constant $C_{\mathcal{Q}}$, if $\tilde{R}_{0}$ is suitably large, such that

$$
\left\langle\mathcal{Q}\left(\eta-E(-1) D_{\frac{1}{\sqrt{t+R^{2}}}} x\right), \eta-E(-1) D_{\frac{1}{\sqrt{t+R^{2}}}} x\right\rangle \geq C_{\mathcal{Q}}|\eta|_{K}^{2}
$$

for every $\eta \in \mathbb{R}^{N+1}$ such that $|\eta|_{K} \geq \frac{r \tilde{R}}{\sqrt{t+R^{2}}}$. Thus

$$
\begin{aligned}
w(x, t) & \leq c_{0} \int_{|\eta|_{K} \geq \frac{r \tilde{R}}{\sqrt{t+R^{2}}}} \exp \left(-C_{\mathcal{Q}} c^{+}|\eta|_{K}^{2}\right) d \eta \\
& \leq c_{0}\left(\int_{\mathbb{R}^{N}} \exp \left(-\frac{1}{2} C_{\mathcal{Q}} c^{+}|\eta|_{K}^{2}\right) d \eta\right) \exp \left(-\frac{r^{2} C_{\mathcal{Q}} c^{+} \tilde{R}^{2}}{2\left(t+R^{2}\right)}\right) .
\end{aligned}
$$

The lemma now follows if we let

$$
C_{0}=c_{0} \int_{\mathbb{R}^{N}} \exp \left(-\frac{1}{2} C_{\mathcal{Q}} c^{+}|\eta|_{K}^{2}\right) d \eta \quad \text { and } \quad C_{1}=\frac{r^{2} C_{\mathcal{Q}} c^{+}}{2 R^{2}} .
$$

\section{Proof of the main theorems}

In this section we prove Theorems 1.5, 1.6 and 1.7. In the following we always assume hypotheses $\mathbf{H 1 - 4}$. Recall that $\mathbf{c}_{\alpha}$ was introduced in (1.21).

Definition 3.1 Let $\Omega \subset \mathbb{R}^{N+1}$ be a given domain, $k \in\{0,1,2\}, \alpha \in(0,1]$ and $M_{1}, M_{2}, M_{3}$ be three positive constants. Let $\psi \in C_{K}^{k, \alpha}(\Omega), g \in C^{0}(\bar{\Omega}), g \geq \psi$ on $\partial_{P} \Omega$, and let $u$ be a strong solution to problem (1.2). Then, for $k \in\{0,1,2\}$ we say that $(u, g, f, \psi)$ belongs to the class $\mathscr{P}_{k}\left(\Omega, \alpha, \mathbf{c}_{\alpha}, M_{1}, M_{2}, M_{3}\right)$ if

$$
\|u\|_{L^{\infty}(\Omega)} \leq M_{1}, \quad\|f\|_{C_{K}^{0, \alpha}(\Omega)} \leq M_{2}, \quad\|\psi\|_{C_{K}^{k, \alpha}(\Omega)} \leq M_{3} .
$$

The proofs of Theorems 1.5, 1.6 and 1.7 are based on certain blow-up arguments. In particular, we introduce, for $r>0$, the blow-up of a function $v \in C^{0}(\Omega)$ as

$$
v^{r}(x, t):=v\left(\delta_{r}(x, t)\right)
$$

whenever $\delta_{r}(x, t) \in \Omega$. A direct computation shows that

$$
L v=f \text { in } \Omega \text { if and only if } L_{r} v^{r}=r^{2} f^{r} \text { in } \delta_{1 / r} \Omega,
$$

where

$$
L_{r}=\sum_{i, j=1}^{m} a_{i j}^{r} \partial_{x_{i} x_{j}}+\sum_{i=1}^{m} r b_{i}^{r} \partial_{x_{i}}+\sum_{i, j=1}^{N} b_{i j} x_{i} \partial_{x_{j}}-\partial_{t} .
$$




\subsection{Optimal interior regularity: proof of Theorems 1.5, 1.6 and 1.7}

To prove Theorems 1.5, 1.6 and 1.7 we first prove the following three lemmas.

Lemma 3.2 Let $\alpha \in(0,1]$ and let $M_{1}, M_{2}, M_{3}$ be positive constants. Assume that

$$
(u, g, f, \psi) \in \mathscr{P}_{0}\left(Q^{-}, \alpha, \mathbf{c}_{\alpha}, M_{1}, M_{2}, M_{3}\right) \quad \text { and } \quad u(0,0)=\psi(0,0)=0 .
$$

Then there exists $c=c\left(N, \lambda, \alpha, \mathbf{c}_{\alpha}, M_{1}, M_{2}, M_{3}\right)$ such that

$$
\left.\sup _{Q_{r}^{-}}|u| \leq c r^{\alpha}, \quad r \in\right] 0,1[.
$$

Lemma 3.3 Let $\alpha \in(0,1]$ and let $M_{1}, M_{2}, M_{3}$ be positive constants. Assume that

$$
(u, g, f, \psi) \in \mathscr{P}_{1}\left(Q^{-}, \alpha, \mathbf{c}_{\alpha}, M_{1}, M_{2}, M_{3}\right) \quad \text { and } \quad u(0,0)=\psi(0,0)=0 .
$$

Then there exists $c=c\left(N, \lambda, \alpha, \mathbf{c}_{\alpha}, M_{1}, M_{2}, M_{3}\right)$ such that

$$
\left.\sup _{Q_{r}^{-}}\left|u(x, t)-\sum_{i=1}^{m} \partial_{x_{i}} \psi(0,0) x_{i}\right| \leq c r^{1+\alpha}, \quad r \in\right] 0,1[\text {. }
$$

Lemma 3.4 Let $\alpha \in(0,1]$ and let $M_{1}, M_{2}, M_{3}$ be positive constants. Assume that

$$
(u, g, f, \psi) \in \mathscr{P}_{2}\left(Q^{-}, \alpha, \mathbf{c}_{\alpha}, M_{1}, M_{2}, M_{3}\right) \quad \text { and } \quad u(0,0)=\psi(0,0)=0 .
$$

Then there exists $c=c\left(N, \lambda, \alpha, \mathbf{c}_{\alpha}, M_{1}, M_{2}, M_{3}\right)$ such that

$$
\left.\sup _{Q_{r}^{-}}|u-\psi| \leq c r^{2}, \quad r \in\right] 0,1[.
$$

The statements of the previous lemmas are structurally the same. We set

$$
S_{k}(u)=\sup _{Q_{2^{-k}}^{-}}|u|
$$

To prove Lemma 3.2 and Lemma 3.3 we intend to prove that there exists a positive $\tilde{c}=$ $\tilde{c}\left(N, \lambda, \alpha, \mathbf{c}_{\alpha}, M_{1}, M_{2}, M_{3}\right)$ such that, for all $k \in \mathbb{N}$,

$$
S_{k+1}(u-F) \leq \max \left(\frac{\tilde{c}}{2^{(k+1) \gamma}}, \frac{S_{k}(u-F)}{2^{\gamma}}, \frac{S_{k-1}(u-F)}{2^{2 \gamma}}, \ldots, \frac{S_{0}(u-F)}{2^{(k+1) \gamma}}\right),
$$

where $F$ and $\gamma$ are determined as follows:

$\diamond F \equiv 0$ and $\gamma=\alpha$ in Lemma 3.2 ,

$\diamond F(x, t)=\sum_{i=1}^{m} \partial_{x_{i}} \psi(0,0) x_{i}$ and $\gamma=1+\alpha$ in Lemma 3.3 . 
Indeed, if (3.5) holds then we see, by a simple iteration argument, that

$$
S_{k}(u-F) \leq \frac{\tilde{c}}{2^{k \gamma}}
$$

and Lemma 3.2 and Lemma 3.3 follow.

Proof of Lemma 3.2 To prove $(3.5)$ with $F=0$ and $\gamma=\alpha$, we assume that

$$
(u, g, f, \psi) \in \mathscr{P}_{0}\left(Q^{-}, \alpha, \mathbf{c}_{\alpha}, M_{1}, M_{2}, M_{3}\right) .
$$

We divide the argument into three steps.

Step 1 (Setting up the argument by contradiction). We first note that

$$
u(x, t) \geq \psi(x, t)=\psi(x, t)-\psi(0,0) \geq-M_{3}\|(x, t)\|_{K}^{\alpha}, \quad(x, t) \in Q^{-} .
$$

Assume that (3.5) is false. Then for every $j \in \mathbb{N}$, there exists a positive integer $k_{j}$ and $\left(u_{j}, g_{j}, f_{j}, \psi_{j}\right) \in \mathscr{P}_{0}\left(Q^{-}, \alpha, \mathbf{c}_{\alpha}, M_{1}, M_{2}, M_{3}\right)$ such that

$$
S_{k_{j}+1}\left(u_{j}\right)>\max \left(\frac{j M_{3}}{2^{\left(k_{j}+1\right) \alpha}}, \frac{S_{k_{j}}\left(u_{j}\right)}{2^{\alpha}}, \frac{S_{k_{j}-1}\left(u_{j}\right)}{2^{2 \alpha}}, \ldots, \frac{S_{0}\left(u_{j}\right)}{2^{\left(k_{j}+1\right) \alpha}}\right) .
$$

Using the definition in (3.4) we see that there exists $\left(x_{j}, t_{j}\right)$ in the closure of $Q_{2^{-k_{j}-1}}^{-}$such that $\left|u_{j}\left(x_{j}, t_{j}\right)\right|=S_{k_{j}+1}\left(u_{j}\right)$ for every $j \geq 1$. Moreover from (3.6) it follows that $u_{j}\left(x_{j}, t_{j}\right)>0$. Using (3.7) we can conclude, as $\left|u_{j}\right| \leq M_{1}$, that $j 2^{-\alpha k_{j}}$ is bounded and hence that $k_{j} \rightarrow \infty$ as $j \rightarrow \infty$.

Step 2 (Constructing blow-ups). We define $\left(\tilde{x}_{j}, \tilde{t}_{j}\right)=\delta_{2^{k_{j}}}\left(\left(x_{j}, t_{j}\right)\right)$ and $\tilde{u}_{j}: Q_{2^{k_{j}}}^{-} \longrightarrow \mathbb{R}$ as

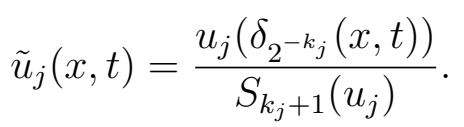

Note that $\left(\tilde{x}_{j}, \tilde{t}_{j}\right)$ belongs to the closure of $Q_{1 / 2}^{-}$and

$$
\tilde{u}_{j}\left(\tilde{x}_{j}, \tilde{t}_{j}\right)=1 \text {. }
$$

Moreover we let $\tilde{L}_{j}=L_{2^{-k_{j}}}($ cf. $(3.3))$ and

$$
\tilde{f}_{j}(x, t)=2^{-2 k_{j}} \frac{f_{j}\left(\delta_{2^{-k_{j}}}(x, t)\right)}{S_{k_{j}+1}\left(u_{j}\right)}, \quad \tilde{g}_{j}(x, t)=\frac{g_{j}\left(\delta_{2^{-k_{j}}}(x, t)\right)}{S_{k_{j}+1}\left(u_{j}\right)}, \quad \tilde{\psi}_{j}(x, t)=\frac{\psi_{j}\left(\delta_{2^{-k_{j}}}(x, t)\right)}{S_{k_{j}+1}\left(u_{j}\right)}
$$

whenever $(x, t) \in Q_{2^{k_{j}}}^{-}$. Then, using (3.2) we see that

$$
\begin{cases}\max \left\{\tilde{L}_{j} \tilde{u}_{j}-\tilde{f}_{j}, \tilde{\psi}_{j}-\tilde{u}_{j}\right\}=0, & \text { in } Q_{2^{k_{j}}}^{-} \\ \tilde{u}_{j}=\tilde{g}_{j}, & \text { on } \partial_{P} Q_{2^{k_{j}}}^{-}\end{cases}
$$

For any $l \in \mathbb{N}$ we have that

$$
\sup _{Q_{2^{l}}^{-}}\left|\tilde{u}_{j}\right|=\frac{S_{k_{j}-l}\left(u_{j}\right)}{S_{k_{j}+1}\left(u_{j}\right)} \leq 2^{(l+1) \alpha} \text { whenever } k_{j}>l .
$$


In particular, we can conclude that

$$
\left(\tilde{u}_{j}, \tilde{u}_{j}, \tilde{f}_{j}, \tilde{\psi}_{j}\right) \in \mathscr{P}_{0}\left(Q_{2^{l}}^{-}, \alpha, \mathbf{c}_{\alpha}, \tilde{M}_{1}^{j}, \tilde{M}_{2}^{j}, \tilde{M}_{3}^{j}\right)
$$

where now $\mathscr{P}_{0}$ is the class related to operator $\tilde{L}_{j}$ and, by (3.10) and (3.11), we have

$$
\tilde{M}_{1}^{j} \leq 2^{(l+1) \alpha}, \quad \tilde{M}_{2}^{j} \leq 2^{-2 k_{j}} \frac{M_{2}}{S_{k_{j}+1}\left(u_{j}\right)}, \quad \tilde{M}_{3}^{j} \leq 2^{\alpha\left(l-k_{j}\right)} \frac{M_{3}}{S_{k_{j}+1}\left(u_{j}\right)} .
$$

Moreover, using (3.7) we see that

$$
\lim _{j \rightarrow \infty} \tilde{M}_{2}^{j}=\lim _{j \rightarrow \infty} \tilde{M}_{3}^{j}=0
$$

Step 3 (Completing the argument by contradiction). In the following we let $l \in \mathbb{N}$ be a suitable large degree of freedom to be specified later. We consider $j_{0} \in \mathbb{N}$ such that $k_{j}>2^{l}$ for $j \geq j_{0}$. We let $\hat{g}_{j}$ denote the boundary values of $\tilde{u}_{j}$ on $\partial_{P} Q_{2^{l}}^{-}$and we let $v_{j}$ and $\tilde{v}_{j}$ be such that

$$
\left\{\begin{array} { l l } 
{ \tilde { L } _ { j } v _ { j } = \| \tilde { f } _ { j } \| _ { L ^ { \infty } ( Q _ { 2 ^ { l } } ^ { - } ) } } & { \text { in } Q _ { 2 ^ { l } } ^ { - } , } \\
{ v _ { j } = \hat { g } _ { j } } & { \text { on } \partial _ { P } Q _ { 2 ^ { l } } ^ { - } , }
\end{array} \quad \left\{\begin{array}{ll}
\tilde{L}_{j} \tilde{v}_{j}=-\left\|\tilde{f}_{j}\right\|_{L^{\infty}\left(Q_{2^{l}}\right)} & \text { in } Q_{2^{l}}^{-}, \\
\tilde{v}_{j}=\max \left\{\hat{g}_{j}, \tilde{M}_{3}^{j}\right\} & \text { on } \partial_{P} Q_{2^{l}}^{-} .
\end{array}\right.\right.
$$

We first prove that

$$
v_{j} \leq \tilde{u}_{j} \leq \tilde{v}_{j} \text { in } Q_{2^{l}}^{-} .
$$

The first inequality in (3.16) follows from the comparison principle. To prove the second one, we first note that $\left\|\tilde{\psi}_{j}\right\|_{\infty} \leq \tilde{M}_{3}^{j}$ and then by the maximum principle $\tilde{v}_{j} \geq \tilde{\psi}_{j}$ in $Q_{2^{l}}^{-}$. Furthermore

$$
\tilde{L}_{j}\left(\tilde{v}_{j}-\tilde{u}_{j}\right)=-\left\|\tilde{f}_{j}\right\|_{L^{\infty}\left(Q_{2^{l}}^{-}\right)}+\tilde{f}_{j} \leq 0 \quad \text { in } \quad \Omega:=Q_{2^{l}}^{-} \cap\left\{(x, t): \tilde{u}_{j}(x, t)>\tilde{\psi}_{j}(x, t)\right\},
$$

and $\tilde{v}_{j} \geq \tilde{u}_{j}$ on $\partial \Omega$. Hence, the second inequality in (3.16) follows from the maximum principle.

We remark that, since $\tilde{u}_{j} \geq \tilde{\psi}_{j}$ by $(3.12)$, we can conclude that $\hat{g}_{j} \geq-\tilde{M}_{3}^{j}$ in $Q_{2^{l}}^{-}$. Hence, using the maximum principle we have, for any positive $T$,

$$
\tilde{v}_{j}(x, t)-v_{j}(x, t) \leq\left(\max \left\{0, \tilde{M}_{3}^{j}-\hat{g}_{j}\right\}+2 T\left\|\tilde{f}_{j}\right\|_{L^{\infty}\left(Q_{2^{l}}^{-}\right.}\right) \leq 2\left(\tilde{M}_{3}^{j}+T \tilde{M}_{2}^{j}\right)
$$

whenever $(x, t) \in Q_{2^{l}}^{-}(0,0, T)$. In particular, we can conclude that

$$
\left\|\tilde{v}_{j}\right\|_{L^{\infty}\left(Q_{2^{l}}^{-}\right)} \leq \max \left\{2^{(l+1) \alpha}, \tilde{M}_{3}^{j}\right\}+2^{2 l} \tilde{M}_{2}^{j}
$$

We claim that there exists a positive constant $C$ such that

$$
\tilde{v}_{j}(x, t) \geq C \quad \text { for every }(x, t) \in Q_{1 / 2}^{-}, j \geq j_{0} \text {. }
$$

Once this claim is prove it follows from from (3.17) and (3.16) that

$$
\tilde{u}_{j}(0,0) \geq v_{j}(0,0) \geq \tilde{v}_{j}(0,0)-2\left(\tilde{M}_{3}^{j}+T \tilde{M}_{2}^{j}\right) \geq C-2\left(\tilde{M}_{3}^{j}+T \tilde{M}_{2}^{j}\right)
$$


and hence, by (3.14), that $\tilde{u}_{j}(0,0)>0$ for $j$ suitably large. This then contradicts the assumption that $\tilde{u}_{j}(0,0)=\tilde{\psi}_{j}(0,0)=0$. Hence our original assumption was incorrect and the proof of the lemma is complete.

In the proof of (3.19) we will use of Lemma 2.6 and 2.7 with $T=1, R=1 / 2$ and

$$
\tilde{R}=\left(\frac{2^{l}}{c}\right)^{\frac{1}{2 \kappa+1}}
$$

where $c$ is the constant in Lemma 2.6. We write $\tilde{v}_{j}=w_{j}+\tilde{w}_{j}+\hat{w}_{j}$ on $Q_{\tilde{R}}^{-}(0,0,1)$ where

$$
\begin{aligned}
& \left\{\begin{array} { l l } 
{ \tilde { L } _ { j } w _ { j } = 0 } & { \text { in } Q _ { \tilde { R } } ^ { - } ( 0 , 0 , 1 ) , } \\
{ w _ { j } = 0 } & { \text { on } \partial _ { P } ^ { + } Q _ { \tilde { R } } ^ { - } ( 0 , 0 , 1 ) , } \\
{ w _ { j } = \tilde { v } _ { j } } & { \text { on } \partial _ { P } ^ { - } Q _ { \tilde { R } } ^ { - } ( 0 , 0 , 1 ) , }
\end{array} \quad \left\{\begin{array}{ll}
\tilde{L}_{j} \tilde{w}_{j}=0 & \text { in } Q_{\tilde{R}}^{-}(0,0,1), \\
\tilde{w}_{j}=\tilde{v}_{j} & \text { on } \partial_{P}^{+} Q_{\tilde{R}}^{-}(0,0,1), \\
\tilde{w}_{j}=0 & \text { on } \partial_{P}^{-} Q_{\tilde{R}}^{-}(0,0,1),
\end{array}\right.\right. \\
& \begin{cases}\tilde{L}_{j} \hat{w}_{j}=-\left\|\tilde{f}_{j}\right\|_{L^{\infty}\left(Q_{2^{l}}^{-}\right)} & \text {in } Q_{\tilde{R}}^{-}(0,0,1), \\
\hat{w}_{j}=0 & \text { on } \partial_{P} Q_{\tilde{R}}^{-}(0,0,1) .\end{cases}
\end{aligned}
$$

where

$$
\partial_{P}^{+} Q_{\tilde{R}}^{-}(0,0, T)=\partial_{P} Q_{\tilde{R}}^{-}(0,0, T) \cap\{t>-T\}, \quad \partial_{P}^{-} Q_{\tilde{R}}^{-}(0,0, T)=\partial_{P} Q_{\tilde{R}}^{-}(0,0, T) \cap\{t=-T\} .
$$

By the maximum principle we easily see that

$$
0 \leq \hat{w}_{j}(x, t) \leq(t+1)\left\|\tilde{f}_{j}\right\|_{L^{\infty}\left(Q_{2^{l}}^{-}\right)} \leq \tilde{M}_{2}^{j},
$$

whenever $(x, t) \in Q_{\tilde{R}}^{-}(0,0,1)$. Hence, as $\left.t \in\right]-1,0\left[\right.$ we see that $\left|\hat{w}_{j}(x, t)\right| \leq 1 / 4$ in $Q_{\tilde{R}}^{-}(0,0,1)$ if $j$ is sufficiently large. Moreover, by Lemma 2.7 and (3.18)

$$
\sup _{Q_{1 / 2}^{-}}\left|\tilde{w}_{j}\right| \leq C_{0} e^{-C_{1} \tilde{R}^{2}} \sup _{\partial_{P}^{+} Q_{\tilde{R}}^{-}}|v| \leq C_{0} e^{-C_{1} \tilde{R}^{2}}\left(\max \left\{2^{(l+1) \alpha}, \tilde{M}_{3}^{j}\right\}+2^{2 l} \tilde{M}_{2}^{j}\right)
$$

and we note that the right hand side in this inequality tends, by the choice in (3.20), to zero as $l$ goes to infinity. Recalling that $\tilde{v}_{j}\left(\tilde{x}_{j}, \tilde{t}_{j}\right) \geq \tilde{u}_{j}\left(\tilde{x}_{j}, \tilde{t}_{j}\right)=1$, we can conclude that by chosing $l$ suitably large we can ensure that

$$
w_{j}\left(\tilde{x}_{j}, \tilde{t}_{j}\right) \geq \frac{1}{2}, \quad j \geq j_{0} .
$$

Using this and the maximum principle we can conclude that there exists at least one point $\left(\bar{x}_{j}, \bar{t}_{j}\right) \in \partial_{P}^{-} Q_{\tilde{R}}^{-}(0,0,1)$ such that

$$
\tilde{v}_{j}\left(\bar{x}_{j}, \bar{t}_{j}\right)=w_{j}\left(\bar{x}_{j}, \bar{t}_{j}\right) \geq \frac{1}{2}, \quad j \geq j_{0} .
$$

We next write $\tilde{v}_{j}=\check{v}_{j}+\hat{v}_{j}$ where

$$
\left\{\begin{array} { l l } 
{ \tilde { L } _ { j } \check { v } _ { j } = 0 } & { \text { in } Q _ { 2 ^ { l } } ^ { - } ( 0 , 0 , 2 ) , } \\
{ \check { v } _ { j } = \tilde { v } _ { j } } & { \text { on } \partial _ { P } Q _ { 2 ^ { l } } ^ { - } ( 0 , 0 , 2 ) . }
\end{array} \quad \left\{\begin{array}{ll}
\tilde{L}_{j} \hat{v}_{j}=-\left\|\tilde{f}_{j}\right\|_{L^{\infty}\left(Q_{2^{l}}^{-}\right)} & \text {in } Q_{2^{l}}^{-}(0,0,2), \\
\hat{v}_{j}=0 & \text { on } \partial_{P} Q_{2^{l}}^{-}(0,0,2)
\end{array}\right.\right.
$$


As in (3.21), we easily see that $\left|\hat{v}_{j}(x, t)\right| \leq 1 / 4$ in $Q_{2^{l}}^{-}(0,0,2)$ if $j$ is sufficiently large and hence we can conclude that

$$
\check{v}_{j}\left(\bar{x}_{j}, \bar{t}_{j}\right) \geq \frac{1}{4}, \quad j \geq j_{0} .
$$

Using Lemma 2.6 we infer that

$$
\inf _{Q_{1 / 2}^{-}} \check{v}_{j} \geq \frac{1}{4 \tilde{c}} .
$$

Since $\hat{v}_{j} \rightarrow 0$ uniformly on $Q_{2^{l}}^{-}(0,0,2)$ as $j$ goes to infinity, we can conclude that

$$
\inf _{Q_{1 / 2}^{-}} \tilde{v}_{j} \geq \inf _{Q_{1 / 2}^{-}} \check{v}_{j}-\left\|\hat{v}_{j}\right\|_{L^{\infty}} \geq \frac{1}{8 \tilde{c}}
$$

for any suitably large $j$. In particular, this proves (3.19) and hence the proof of Lemma 3.2 is complete.

Proof of Lemma 3.3 We argue as in the proof of Lemma 3.2 to show that (3.5) holds with $F(x, t)=\sum_{i=1}^{m} \partial_{x_{i}} \psi(0,0) x_{i}$ and $\gamma=\alpha+1$. We assume that

$$
(u, g, f, \psi) \in \mathscr{P}_{1}\left(Q^{-}, \alpha, \mathbf{c}_{\alpha}, M_{1}, M_{2}, M_{3}\right) .
$$

We divide the argument into three steps.

Step 1 (Setting up the argument by contradiction). We first note that, since $\psi \in C_{K}^{1, \alpha}$,

$$
u(x, t) \geq \psi(x, t)=\psi(x, t)-\psi(0,0) \geq F(x, t)-M_{3}\|(x, t)\|_{K}^{1+\alpha}, \quad(x, t) \in Q^{-} .
$$

As in the proof of Lemma 3.2 , we assume that (3.5) is false. Then for every $j \in \mathbb{N}$, there exists a positive integer $k_{j}$ and $\left(u_{j}, g_{j}, f_{j}, \psi_{j}\right) \in \mathscr{P}_{1}\left(Q^{-}, \alpha, \mathbf{c}_{\alpha}, M_{1}, M_{2}, M_{3}\right)$ such that

$$
S_{k_{j}+1}\left(u_{j}-F_{j}\right)>\max \left(\frac{j M_{3}}{2^{\left(k_{j}+1\right) \gamma}}, \frac{S_{k_{j}}\left(u_{j}-F_{j}\right)}{2^{\gamma}}, \frac{S_{k_{j}-1}\left(u_{j}-F_{j}\right)}{2^{2 \gamma}}, \ldots ., \frac{S_{0}\left(u_{j}-F_{j}\right)}{2^{\left(k_{j}+1\right) \gamma}}\right),
$$

where $F_{j}(x, t)=\sum_{i=1}^{m} \partial_{x_{i}} \psi_{j}(0,0) x_{i}$. By (3.4) there exists $\left(x_{j}, t_{j}\right)$ in the closure of $Q_{2^{-k_{j}-1}}^{-}$such that $u_{j}\left(x_{j}, t_{j}\right)-F\left(x_{j}, t_{j}\right)=S_{k_{j}+1}\left(u_{j}-F\right)$ for every $j \geq 1$. Moreover, by (3.23) we infer that $k_{j} \rightarrow \infty$ as $j \rightarrow \infty$.

Step 2 (Constructing blow-ups). We define $\left(\tilde{x}_{j}, \tilde{t}_{j}\right)=\delta_{2^{k_{j}}}\left(\left(x_{j}, t_{j}\right)\right)$ and $\tilde{u}_{j}: Q_{2^{k_{j}}}^{-} \longrightarrow \mathbb{R}$ as

$$
\tilde{u}_{j}(x, t)=\frac{\left(u_{j}-F_{j}\right)\left(\delta_{2^{-k_{j}}}(x, t)\right)}{S_{k_{j}+1}\left(u_{j}-F_{j}\right)} .
$$

As before, we have that $\left(\tilde{x}_{j}, \tilde{t}_{j}\right)$ belongs to the closure of $Q_{1 / 2}^{-}$and

$$
\tilde{u}_{j}\left(\tilde{x}_{j}, \tilde{t}_{j}\right)=1
$$


Now we let

$$
\begin{aligned}
\tilde{f}_{j}(x, t) & =2^{-2 k_{j}} \frac{\left(f_{j}-L F_{j}\right)\left(\delta_{2^{-k_{j}}}(x, t)\right)}{S_{k_{j}+1}\left(u_{j}-F_{j}\right)}, \quad \tilde{g}_{j}(x, t)=\frac{\left(g_{j}-F_{j}\right)\left(\delta_{2^{-k_{j}}}(x, t)\right)}{S_{k_{j}+1}\left(u_{j}-F_{j}\right)}, \\
\tilde{\psi}_{j}(x, t) & =\frac{\left(\psi_{j}-F_{j}\right)\left(\delta_{2}-k_{j}(x, t)\right)}{S_{k_{j}+1}\left(u_{j}-F_{j}\right)}
\end{aligned}
$$

whenever $(x, t) \in Q_{2^{k_{j}}}^{-}$. Then, by setting $\tilde{L}_{j}=L_{2^{-k_{j}}}$, we have

$$
\begin{cases}\max \left\{\tilde{L}_{j} \tilde{u}_{j}-\tilde{f}_{j}, \tilde{\psi}_{j}-\tilde{u}_{j}\right\}=0, & \text { in } Q_{2^{k_{j}}}^{-} \\ \tilde{u}_{j}=\tilde{g}_{j}, & \text { on } \partial_{P} Q_{2^{k_{j}}}^{-}\end{cases}
$$

As in the proof of Lemma 3.2 , we have

$$
\left(\tilde{u}_{j}, \tilde{u}_{j}, \tilde{f}_{j}, \tilde{\psi}_{j}\right) \in \mathscr{P}_{1}\left(Q_{2^{l}}^{-}, \alpha, \mathbf{c}_{\alpha}, \tilde{M}_{1}^{j}, \tilde{M}_{2}^{j}, \tilde{M}_{3}^{j}\right)
$$

where $\mathscr{P}_{1}$ is the class related to operator $\tilde{L}_{j}$. However we prove our claim by using only the semi-norm

$$
N_{3}^{j}:=\sum_{i=1}^{m}\left|\partial_{x_{i}} \tilde{\psi}_{j}(0,0)\right|+\sup _{\substack{z, \zeta \in Q^{-} \\ z \neq \zeta}} \frac{\left|\tilde{\psi}_{j}(z)-\tilde{\psi}_{j}(\zeta)-\sum_{i=1}^{m}\left(x_{i}-\xi_{i}\right) \partial_{x_{i}} \tilde{\psi}_{j}(\zeta)\right|}{\left\|\zeta^{-1} \circ z\right\|_{K}^{1+\alpha}}
$$

and remark that $N_{3}^{j}+\left\|\tilde{\psi}_{j}\right\|_{C_{K}^{0, \alpha}\left(Q^{-}\right)} \leq \tilde{M}_{3}^{j}$. Since $L F_{j}(x, t)=\sum_{i=1}^{m} b_{i}(x, t) \partial_{x_{i}} \psi_{j}(0,0)$, we have

$$
\tilde{M}_{1}^{j} \leq 2^{(l+1)(1+\alpha)}, \quad \tilde{M}_{2}^{j} \leq 2^{-2 k_{j}} \frac{M_{2}+\mathbf{c}_{\alpha} M_{3}}{S_{k_{j}+1}\left(u_{j}-F_{j}\right)} .
$$

Furthermore,

$$
N_{3}^{j} \leq 2^{(1+\alpha)\left(l-k_{j}\right)} \frac{M_{3}}{S_{k_{j}+1}\left(u_{j}-F_{j}\right)} .
$$

To see this we note that $\partial_{x_{i}} \tilde{\psi}_{j}(0,0)=0$ and

$$
\begin{aligned}
& \left|\left(\psi_{j}-F_{j}\right)(z)-\left(\psi_{j}-F_{j}\right)(\zeta)-\sum_{i=1}^{m}\left(x_{i}-\xi_{i}\right) \partial_{x_{i}}\left(\psi_{j}-F_{j}\right)(\zeta)\right| \\
& =\mid\left(\psi_{j}(z)-\psi_{j}(\zeta)-\sum_{i=1}^{m}\left(x_{i}-\xi_{i}\right) \partial_{x_{i}} \psi_{j}(\zeta)\right) \\
& -\sum_{i=1}^{m}\left(x_{i}-\xi_{i}-\left(x_{i}-\xi_{i}\right)\right) \partial_{x_{i}} \psi_{j}(0,0) \mid \leq M_{3}\left\|\zeta^{-1} \circ z\right\|_{K}^{1+\alpha} .
\end{aligned}
$$

Therefore, by (3.23), we have

$$
\lim _{j \rightarrow \infty} \tilde{M}_{2}^{j}=\lim _{j \rightarrow \infty} N_{3}^{j}=0
$$

Step 3 (Completing the argument by contradiction). This step is completely analogous to Step 3 in Lemma 3.2 except for the use of $N_{3}^{j}$ in place of $\tilde{M}_{3}^{j}$ and $\gamma$ now equals $1+\alpha$. 
Proof of Lemma 3.4 We first note that we may assume that $\psi \equiv 0$. Indeed, using the fact that $L \psi \in C_{K}^{0, \alpha}$ we can consider $\tilde{u}=u-\psi, \tilde{f}=f-\underset{\tilde{N}}{L}, \tilde{g}=g-\psi$ in $Q^{-}$. In particular, we note that $(\tilde{u}, \tilde{g}, \tilde{f}, 0) \in \mathscr{P}_{2}\left(Q^{-}, \alpha, \mathbf{c}_{\alpha}, \tilde{M}_{1}, \tilde{M}_{2}, 0\right)$ where $\left(\tilde{M}_{1}, \tilde{M}_{2}\right)$ only depends on $M_{1}, M_{2}$ and $M_{3}$. From here on we can now argue as in the proof of the previous two lemmas. In particular, we note that

$$
u(x, t) \geq \psi(x, t)=0, \quad(x, t) \in Q^{-} .
$$

and we assume that $(\overline{3.5})$ is false with $\gamma=2$. Then for every $j \in \mathbb{N}$, there exists a positive integer $k_{j}$ and $\left(u_{j}, g_{j}, f_{j}, 0\right) \in \mathscr{P}_{2}\left(Q^{-}, \alpha, \mathbf{c}_{\alpha}, M_{1}, M_{2}, 0\right)$ such that

$$
S_{k_{j}+1}\left(u_{j}\right)>\max \left(\frac{j}{2^{2\left(k_{j}+1\right)}}, \frac{S_{k_{j}}\left(u_{j}\right)}{2^{2}}, \frac{S_{k_{j}-1}\left(u_{j}\right)}{2^{4}}, \ldots, \frac{S_{0}\left(u_{j}\right)}{2^{2\left(k_{j}+1\right)}}\right) .
$$

By (3.4) there exists $\left(x_{j}, t_{j}\right)$ in the closure of $Q_{2^{-k_{j}-1}}^{-}$such that $u_{j}\left(x_{j}, t_{j}\right)=S_{k_{j}+1}\left(u_{j}\right)$ for every $j \geq 1$ and, by (3.31), $k_{j} \rightarrow \infty$ as $j \rightarrow \infty$. The rest of the proof then follow along the same lines as the proof of Lemma 3.2 .

We next derive the following extensions of Lemmas 3.2, 3.3 and 3.4 .

Lemma 3.5 Let $\alpha \in(0,1]$ and let $M_{1}, M_{2}, M_{3}$ be positive constants. Assume that

$$
(u, g, f, \psi) \in \mathscr{P}_{0}\left(Q, \alpha, \mathbf{c}_{\alpha}, M_{1}, M_{2}, M_{3}\right) \quad \text { and } \quad u(0,0)=\psi(0,0)=0 .
$$

Then there exists $c=c\left(N, \lambda, \alpha, \mathbf{c}_{\alpha}, M_{1}, M_{2}, M_{3}\right)$ such that

$$
\left.\sup _{Q_{r}}|u| \leq c r^{\alpha}, \quad r \in\right] 0,1[.
$$

Lemma 3.6 Let $\alpha \in(0,1]$ and let $M_{1}, M_{2}, M_{3}$ be positive constants. Assume that

$$
(u, g, f, \psi) \in \mathscr{P}_{1}\left(Q, \alpha, \mathbf{c}_{\alpha}, M_{1}, M_{2}, M_{3}\right) \quad \text { and } \quad u(0,0)=\psi(0,0)=0 .
$$

Then there exists $c=c\left(N, \lambda, \alpha, \mathbf{c}_{\alpha}, M_{1}, M_{2}, M_{3}\right)$ such that

$$
\left.\sup _{Q_{r}}\left|u(x, t)-\sum_{i=1}^{m} \partial_{x_{i}} \psi(0,0) x_{i}\right| \leq c r^{1+\alpha}, \quad r \in\right] 0,1[.
$$

Lemma 3.7 Let $\alpha \in(0,1]$ and let $M_{1}, M_{2}, M_{3}$ be positive constants. Assume that

$$
(u, g, f, \psi) \in \mathscr{P}_{2}\left(Q, \alpha, \mathbf{c}_{\alpha}, M_{1}, M_{2}, M_{3}\right) \quad \text { and } \quad u(0,0)=\psi(0,0)=0 .
$$

Then there exists $c=c\left(N, \lambda, \alpha, \mathbf{c}_{\alpha}, M_{1}, M_{2}, M_{3}\right)$ such that

$$
\left.\sup _{Q_{r}}|u-\psi| \leq c r^{2}, \quad r \in\right] 0,1[.
$$

Proof of Lemma 3.5 Using Lemma 3.2 we see that we only have to prove that there exists a constant $c=c\left(N, \lambda, \alpha, \mathbf{c}_{\alpha}, M_{1}, M_{2}, M_{3}\right)$ such that

$$
\sup _{Q_{r}^{+}}|u| \leq c r^{\alpha}, \quad r \in[0,1] .
$$


We set $v=v_{1}+v_{2}+v_{3}$ where

$$
\left\{\begin{array} { l l } 
{ L v _ { 1 } = 0 } & { \text { in } Q ^ { + } , } \\
{ v _ { 1 } = 0 } & { \text { on } \partial _ { P } ^ { + } Q ^ { + } , } \\
{ v _ { 1 } = | u | } & { \text { on } \partial _ { P } ^ { - } Q ^ { + } , }
\end{array} \quad \left\{\begin{array} { l l } 
{ L v _ { 2 } = 0 } & { \text { in } Q ^ { + } , } \\
{ v _ { 2 } = | u | } & { \text { on } \partial _ { P } ^ { + } Q ^ { + } , } \\
{ v _ { 2 } = 0 } & { \text { on } \partial _ { P } ^ { - } Q ^ { + } , }
\end{array} \quad \left\{\begin{array}{ll}
L v_{3}=-\|f\|_{L^{\infty}\left(Q^{+}\right)} & \text {in } Q^{+}, \\
v_{3}=0 & \text { on } \partial_{P} Q^{+},
\end{array}\right.\right.\right.
$$

where

$$
\partial_{P}^{+} Q^{+}=\partial_{P} Q^{+} \cap\{t>0\}, \quad \partial_{P}^{-} Q^{+}=\partial_{P} Q^{+} \cap\{t=0\} .
$$

By the maximum principle we see that $\psi \leq u \leq v$ in $Q^{+}$and hence we only have to prove that

$$
\sup _{Q_{r}^{+}}|v| \leq c r^{\alpha}
$$

for $r$ suitably small.

To continue we first note, using Lemma 3.2 and Corollary 2.4, that

$$
\left|v_{1}(x, t)\right| \leq c \int_{|y|_{K} \leq R_{0}} \Gamma(x, t, y, 0)|y|_{K}^{\alpha} d y \leq c_{\alpha}\|(x, t)\|_{K}^{\alpha},
$$

for some $R_{0}>1$. For any positive $r$ we consider the function $u_{2}(x, t)=v_{2}\left(D_{r} x, r^{2}(t+1)\right)$. By applying Lemma 2.7 with $R=1$ and $\tilde{R}=\frac{1}{r}$ we see that there exist positive constants $C_{0}, C_{1}$, and $R_{0}$ such that

$$
\sup _{Q_{r}^{+}}\left|v_{2}\right|=\sup _{Q^{-}}\left|u_{2}\right| \leq C_{0} \exp \left(-C_{1} \tilde{R}^{2}\right) \sup _{\partial_{P} Q_{\tilde{R}}^{-}\{\{(x, t): t>-1\}}\left|u_{2}\right| \leq C_{0} \exp \left(-\frac{C_{1}}{r^{2}}\right) \sup _{\partial_{P}^{+} Q^{+}}\left|v_{2}\right|
$$

whenever $r \leq \frac{1}{R_{0}}$. Since $v_{2}$ agrees with $|u|$ on $\partial_{P}^{+} Q^{+}$we can conclude that

$$
\left.\left.\sup _{Q_{r}^{+}}\left|v_{2}\right| \leq C_{0} M_{1} \exp \left(-\frac{C_{1}}{r^{2}}\right) \leq c_{2} r^{\alpha}, \quad \text { for every } \quad r \in\right] 0, \frac{1}{R_{0}}\right]
$$

Finally, we have

$$
\left|v_{3}(x, t)\right| \leq\|f\|_{L^{\infty}\left(Q^{+}\right)} \int_{0}^{t} \int_{\mathbb{R}^{N}} \Gamma(x, t, y, s) d y d s \leq t\|f\|_{L^{\infty}\left(Q^{+}\right)} \leq M_{2}\|(x, t)\|_{K}^{2} .
$$

Put together these estimates prove that the claim in (3.33) is true.

Proof of Lemma 3.6 and 3.7. To prove these two lemmas we can use the same argument as in the proof of Lemma 3.5. In particular, we now apply Corollary 2.4 with $\gamma=\alpha+1$ and $\gamma=2$ in the proof of Lemma 3.6 and Lemma 3.7 respectively.

In order to prove Theorems 1.5, 1.6 and 1.7, we introduce some further notations. For $(x, t) \in \Omega$ and $r \in(0,1)$, we introduce the blow-up of a function $v \in C^{0}(\Omega)$ at $(x, t)$ as

$$
v^{r,(x, t)}(y, s):=v\left((x, t) \circ \delta_{r}(y, s)\right)
$$


whenever $(x, t) \circ \delta_{r}(y, s) \in \Omega$, and the corresponding scaled operator,

$$
L_{r}^{(y, s)}=\sum_{i, j=1}^{m} a_{i j}^{r,(x, t)}(y, s) \partial_{y_{i} y_{j}}+\sum_{i=1}^{m} r b_{i}^{r,(x, t)}(y, s) \partial_{y_{i}}+\sum_{i, j=1}^{N} b_{i j} y_{i} \partial_{y_{j}}-\partial_{s} .
$$

Proof of Theorem 1.5 We fix $R \in] 0,1\left[\right.$ such that $Q_{2 R} \subseteq Q_{2 R C_{1}}(\hat{x}, \hat{t}) \subseteq Q$ for every $(\hat{x}, \hat{t}) \in Q_{2 R}$, where $C_{1}$ is the constant in (1.19). Let $\mathscr{F}=\bar{Q}_{2 R} \cap\{(x, t): u(x, t)=\psi(x, t)\}$. If $\mathscr{F}$ is empty then the result is a direct consequence of Theorem 2.1. We aim to prove that there exists a positive constant $\hat{c}=\hat{c}\left(N, \lambda, \alpha, \mathbf{c}_{\alpha}, M_{1}, M_{2}, M_{3}\right)$ such that

$$
\sup _{\substack{(x, t),(\hat{x}, \hat{t}) \in Q_{R} \\(x, t) \neq(\hat{x}, \hat{t})}} \frac{|u(x, t)-u(\hat{x}, \hat{t})|}{\left\|(\hat{x}, \hat{t})^{-1} \circ(x, t)\right\|_{K}^{\alpha}} \leq \hat{c} .
$$

If $(\hat{x}, \hat{t}) \in Q_{2 R} \cap \mathscr{F}$, then we apply Lemma 3.5 and we get

$$
|u(x, t)-u(\hat{x}, \hat{t})| \leq c\left\|(\hat{x}, \hat{t})^{-1} \circ(x, t)\right\|_{K}^{\alpha} \quad \text { for every }(x, t) \in Q_{2 R C_{1}}(\hat{x}, \hat{t}) .
$$

The same result clearly holds whenever $(x, t) \in Q_{R} \cap \mathscr{F}$. To complete the proof of Theorem 1.5 we therefore assume that $(x, t),(\hat{x}, \hat{t}) \in Q_{R} \backslash \mathscr{F}$. Let $r=d_{K}((x, t), \mathscr{F})$ denote the distance from $(x, t)$ to $\mathscr{F}$, as defined in (1.16) and let $(\tilde{x}, \tilde{t}) \in \mathscr{F}$ be such that $r=d_{K}((x, t),(\tilde{x}, \tilde{t}))$. We divide the proof into two cases.

Case 1. Assume $(\hat{x}, \hat{t}) \in Q_{R} \backslash Q_{r / 2}(x, t)$. Then $d_{K}((x, t),(\hat{x}, \hat{t}))>c_{0} r$ for some universal positive constant $c_{0}$. By the triangle inequality (1.15) we have

$$
d_{K}((\hat{x}, \hat{t}),(\tilde{x}, \tilde{t})) \leq c\left(d_{K}((x, t),(\tilde{x}, \tilde{t}))+c d_{K}((x, t),(\hat{x}, \hat{t}))\right) .
$$

Recalling that $d_{K}((x, t),(\tilde{x}, \tilde{t})) \leq \frac{1}{c_{0}} d_{K}((x, t),(\hat{x}, \hat{t}))$, we see, using (3.37), that

$$
\begin{aligned}
|u(x, t)-u(\hat{x}, \hat{t})| & \leq|u(x, t)-u(\tilde{x}, \tilde{t})|+|u(\hat{x}, \hat{t})-u(\tilde{x}, \tilde{t})| \\
& \leq c\left(d_{K}((x, t),(\tilde{x}, \tilde{t}))^{\alpha}+d_{K}((\hat{x}, \hat{t}),(\tilde{x}, \tilde{t}))^{\alpha}\right) \leq \hat{c}\left\|(\hat{x}, \hat{t})^{-1} \circ(x, t)\right\|_{K}^{\alpha},
\end{aligned}
$$

for some positive constant $\hat{c}$ only depending on $c_{0}$ and on the constant $c$ in (1.15).

Case 2. Assume $(\hat{x}, \hat{t}) \in Q_{r / 2}(x, t)$. We set

$$
v(\hat{x}, \hat{t})=u(\hat{x}, \hat{t})-u(\tilde{x}, \tilde{t}) .
$$

¿From (3.38) it follows that there exists a positive universal constant $c_{1}$ such that $|v(\hat{x}, \hat{t})| \leq c_{1} r^{\alpha}$. Then the function $w(y, s)=\frac{v^{r,(x, t)}(y, s)}{r^{\alpha}}$ satisfies

$$
|w(y, s)| \leq c_{1} \quad \text { and } \quad L^{r,(x, t)} w(y, s)=r^{2-\alpha} f^{r,(x, t)}(y, s) \quad \text { for }(y, s) \in Q_{1 / 2} .
$$

Hence, by the Schauder estimates in Theorem 2.1, we have

$$
|w(y, s)-w(0,0)| \leq \hat{c}_{1}\|(y, s)\|_{K}^{\alpha}
$$


for some positive constant $\hat{c}_{1}$, that only depends on $c_{1}$ and on the constants in Theorem 2.1. Then, for $(\hat{x}, \hat{t})=(x, t) \circ \delta_{r}(y, s)$, we get

$$
|u(\hat{x}, \hat{t})-u(x, t)| \leq \hat{c}_{1}\left\|(x, t)^{-1} \circ(\hat{x}, \hat{t})\right\|_{K}^{\alpha},
$$

and this completes the proof of Theorem 1.5 .

Proof of Theorem 1.6 Let $R$ and $\mathscr{F}$ be as in the proof of Theorem 1.5: we still assume that $\mathscr{F} \neq \emptyset$ otherwise the thesis is a trivial consequence of Theorem 2.1. In Theorem 1.5 we have already proved that $\|u\|_{C_{K}^{0, \alpha}\left(Q_{R}\right)} \leq \hat{c}$. We next prove that $\left\|\partial_{x_{i}} u\right\|_{C_{K}^{0, \alpha}\left(Q_{R}\right)} \leq \hat{c}$ for $i=1, \ldots, m$. From Lemma 3.6, we see that $\partial_{x_{i}} u(x, t)=\partial_{x_{i}} \psi(x, t)$ for every $(x, t) \in \mathscr{F}$. Using the same notations as in the proof of Theorem [1.5, we consider $(x, t) \in Q_{R} \backslash \mathscr{F}$ and we let $(\tilde{x}, \tilde{t}) \in \mathscr{F}$ such that $r:=d_{K}((x, t), \mathscr{F})=d_{K}((x, t),(\tilde{x}, \tilde{t}))$. Furthermore we define

$$
v(\hat{x}, \hat{t})=u(\hat{x}, \hat{t})-u(\tilde{x}, \tilde{t})-\sum_{i=1}^{m} \partial_{x_{i}} u(\tilde{x}, \tilde{t})\left(\hat{x}_{i}-\tilde{x}_{i}\right) .
$$

Then, by applying Lemma 3.6 in the cylinder $Q_{2 R C_{1}}(\tilde{x}, \tilde{t})$, we see that

$$
|v(\hat{x}, \hat{t})| \leq c_{2} d_{K}((\hat{x}, \hat{t}),(\tilde{x}, \tilde{t}))^{1+\alpha}, \quad(\hat{x}, \hat{t}) \in Q_{2 R},
$$

for some positive universal constant $c_{2}$. We next prove that

$$
\sup _{(\hat{x}, \hat{t}) \in Q_{R} \backslash \mathscr{F},(\tilde{x}, \tilde{t}) \in Q_{R} \cap \mathscr{F}}\left|\partial_{x_{i}} u(\hat{x}, \hat{t})-\partial_{x_{i}} u(\tilde{x}, \tilde{t})\right| \leq \hat{c}_{3} d_{K}((\hat{x}, \hat{t}),(\tilde{x}, \tilde{t}))^{\alpha}, \quad i=1, \ldots, m .
$$

Assume $d_{K}((\hat{x}, \hat{t}),(\tilde{x}, \tilde{t})) \leq 2 \hat{r}$. In this case the function

$$
w(y, s)=\frac{v^{\hat{r},(\hat{x}, \hat{t})}(y, s)}{\hat{r}^{1+\alpha}}
$$

satisfies

$$
|w| \leq c_{2}^{\prime} \quad \text { and } \quad L^{\hat{r},(\hat{x}, \hat{t})} w=\hat{r}^{1-\alpha}\left(f^{\hat{r},(\hat{x}, \hat{t})}+\sum_{i=1}^{m} b_{i}^{\hat{r},(\hat{x}, \hat{t})} \partial_{x_{i}} \psi(\tilde{x}, \tilde{t})\right) \quad \text { in } Q_{1 / 2} .
$$

Hence, by the Schauder estimates in Theorem 2.1,

$$
\left\|\partial_{y_{i}} w\right\|_{C_{K}^{0, \alpha}\left(Q_{R}\right)} \leq \hat{c}_{2}, \quad \text { for } i=1, \ldots, m
$$

In particular, since

$$
\partial_{y_{i}} w(0,0)=\frac{\partial_{x_{i}} u(\hat{x}, \hat{t})-\partial_{x_{i}} \psi(\tilde{x}, \tilde{t})}{\hat{r}^{\alpha}}
$$

we have

$$
\left|\partial_{x_{i}} u(\hat{x}, \hat{t})-\partial_{x_{i}} u(\tilde{x}, \tilde{t})\right| \leq \hat{c}_{2} \hat{r}^{\alpha} \leq \hat{c}_{2} d_{K}((\hat{x}, \hat{t}),(\tilde{x}, \tilde{t}))^{\alpha} \text {. }
$$

This completes the proof of (3.40) in case $d_{K}((\hat{x}, \hat{t}),(\tilde{x}, \tilde{t})) \leq 2 \hat{r}$. Moreover, since (3.44) holds for $(\hat{x}, \hat{t})=(x, t)($ with $r=\hat{r})$ we also have

$$
\sup _{Q_{R}}\left|\partial_{x_{i}} u\right| \leq \hat{c}_{2}+\sup _{Q_{R}}\left|\partial_{x_{i}} \psi\right|, \quad i=1, \ldots, m,
$$


and hence we have established the appropriate estimate on the supremum of $\left|\partial_{x_{i}} u\right|$. To prove $(3.40)$ in case $d_{K}((\hat{x}, \hat{t}),(\tilde{x}, \tilde{t})) \geq 2 \hat{r}$ we let $(\bar{x}, \bar{t}) \in \mathscr{F}$ be such that $\hat{r}:=d_{K}((\hat{x}, \hat{t}), \mathscr{F})=$ $d_{K}((\hat{x}, \hat{t}),(\bar{x}, \bar{t}))$. Then, by the triangular inequality, we see that

$$
d_{K}((\bar{x}, \bar{t}),(\tilde{x}, \tilde{t})) \leq c\left(d_{K}((\bar{x}, \bar{t}),(\hat{x}, \hat{t}))+d_{K}((\hat{x}, \hat{t}),(\tilde{x}, \tilde{t}))\right) \leq c_{3} d_{K}((\hat{x}, \hat{t}),(\tilde{x}, \tilde{t})) .
$$

Hence, using (3.43) it follows that

$$
\begin{aligned}
\left|\partial_{x_{i}} u(\hat{x}, \hat{t})-\partial_{x_{i}} u(\tilde{x}, \tilde{t})\right| & \leq\left|\partial_{x_{i}} u(\hat{x}, \hat{t})-\partial_{x_{i}} u(\bar{x}, \bar{t})\right|+\left|\partial_{x_{i}} \psi(\bar{x}, \bar{t})-\partial_{x_{i}} \psi(\tilde{x}, \tilde{t})\right| \\
& \leq \hat{c}_{2} d_{K}((\hat{x}, \hat{t}),(\bar{x}, \bar{t}))^{\alpha}+\|\psi\|_{C_{K}^{1, \alpha}} d_{K}((\bar{x}, \bar{t}),(\tilde{x}, \tilde{t}))^{\alpha} \\
& \leq \hat{c}_{3} d_{K}((\hat{x}, \hat{t}),(\tilde{x}, \tilde{t})) .
\end{aligned}
$$

This concludes the proof of (3.40).

We next complete the proof of the Hölder continuity of $\partial_{x_{i}} u$. We divide the proof into two cases.

Case 1. Assume $(\hat{x}, \hat{t}) \in Q_{R} \backslash Q_{r / 2}(x, t)$. Then by the triangle inequality (3.38) and (3.40) we have

$$
\begin{aligned}
\left|\partial_{x_{i}} u(x, t)-\partial_{x_{i}} u(\hat{x}, \hat{t})\right| & \leq\left|\partial_{x_{i}} u(x, t)-\partial_{x_{i}} u(\tilde{x}, \tilde{t})\right|+\left|\partial_{x_{i}} u(\hat{x}, \hat{t})-\partial_{x_{i}} u(\tilde{x}, \tilde{t})\right| \\
& \leq \hat{c}_{3}\left(d_{K}((x, t),(\tilde{x}, \tilde{t}))^{\alpha}+d_{K}((\hat{x}, \hat{t}),(\tilde{x}, \tilde{t}))^{\alpha}\right) \\
& \leq \hat{c}_{4}\left\|(\hat{x}, \hat{t})^{-1} \circ(x, t)\right\|_{K}^{\alpha} .
\end{aligned}
$$

Case 2. Assume $(\hat{x}, \hat{t}) \in Q_{r / 2}(x, t)$. We consider the function $w$ in $(3.41)$ with $(\hat{x}, \hat{t})=(x, t)$. From (3.43) it follows that

$$
\left|\partial_{x_{i}} u\left((x, t) \circ \delta_{r}(y, s)\right)-\partial_{x_{i}} u(x, t)\right|=r^{\alpha}\left|\partial_{y_{i}} w(y, s)-\partial_{y_{i}} w(0,0)\right| \leq \hat{c}_{2}\left(r\|(y, s)\|_{K}\right)^{\alpha} .
$$

This concludes the estimate of $\left\|\partial_{x_{i}} u\right\|_{C_{K}^{0, \alpha}\left(Q_{R}\right)}$.

We finally prove, whenever $(x, t),(\hat{x}, \hat{t}) \in Q_{R}$, that

$$
\left|u(x, t)-u(\hat{x}, \hat{t})-\sum_{i=1}^{m} \partial_{x_{i}} u(\hat{x}, \hat{t})\left(x_{i}-\hat{x}_{i}\right)\right| \leq c\left\|(x, t)^{-1} \circ(\hat{x}, \hat{t})\right\|_{K}^{1+\alpha},
$$

for some constant $c$ only dependent on $\alpha$, the operator $L$ and the data $f, g$ and $\psi$. We note that if $(\hat{x}, \hat{t}) \in \mathscr{F}$ then the inequality in (3.45) follows from Lemma 3.6. Moreover, if $(x, t) \in \mathscr{F}$ then, by Lemma 3.6 and (3.40), we have

$$
\begin{aligned}
& \left|u(x, t)-u(\hat{x}, \hat{t})-\sum_{i=1}^{m} \partial_{x_{i}} u(\hat{x}, \hat{t})\left(x_{i}-\hat{x}_{i}\right)\right| \leq\left|u(\hat{x}, \hat{t})-u(x, t)-\sum_{i=1}^{m} \partial_{x_{i}} u(x, t)\left(\hat{x}_{i}-x_{i}\right)\right| \\
& +\sum_{i=1}^{m}\left|\partial_{x_{i}} u(x, t)-\partial_{x_{i}} u(\hat{x}, \hat{t})\right|\left|\hat{x}_{i}-x_{i}\right| \leq c\left\|(x, t)^{-1} \circ(\hat{x}, \hat{t})\right\|_{K}^{1+\alpha} .
\end{aligned}
$$

To complete the proof of (3.45) we can therefore assume that $(x, t),(\hat{x}, \hat{t}) \in Q_{R} \backslash \mathscr{F}$ and we let, as before, $r=d_{K}((x, t), \mathscr{F})$ and $(\tilde{x}, \tilde{t}) \in \mathscr{F}$ be such that $r=d_{K}((x, t),(\tilde{x}, \tilde{t}))$. We divide the 
proof into two cases.

Case 1. Assume $(\hat{x}, \hat{t}) \in Q_{R} \backslash Q_{r / 2}(x, t)$. Then by the triangle inequality (3.38) and (3.40) we have

$$
\begin{aligned}
& \left|u(x, t)-u(\hat{x}, \hat{t})-\sum_{i=1}^{m} \partial_{x_{i}} u(\hat{x}, \hat{t})\left(x_{i}-\hat{x}_{i}\right)\right| \leq\left|u(x, t)-u(\tilde{x}, \tilde{t})-\sum_{i=1}^{m} \partial_{x_{i}} u(\tilde{x}, \tilde{t})\left(x_{i}-\tilde{x}_{i}\right)\right|+ \\
& u(\tilde{x}, \tilde{t})-u(\hat{x}, \hat{t})-\sum_{i=1}^{m} \partial_{x_{i}} u(\hat{x}, \hat{t})\left(\tilde{x}_{i}-\hat{x}_{i}\right)\left|+\sum_{i=1}^{m}\right| \partial_{x_{i}} u(\tilde{x}, \tilde{t})-\partial_{x_{i}} u(\hat{x}, \hat{t})|| x_{i}-\tilde{x}_{i} \mid \\
& \leq c\left(d_{K}((x, t),(\tilde{x}, \tilde{t}))^{1+\alpha}+d_{K}((\hat{x}, \hat{t}),(\tilde{x}, \tilde{t}))^{1+\alpha}+d_{K}((x, t),(\tilde{x}, \tilde{t})) d_{K}((\hat{x}, \hat{t}),(\tilde{x}, \tilde{t}))^{\alpha}\right) \\
& \leq \hat{c}_{5}\left\|(\hat{x}, \hat{t})^{-1} \circ(x, t)\right\|_{K}^{1+\alpha} .
\end{aligned}
$$

Case 2. Assume $(\hat{x}, \hat{t}) \in Q_{r / 2}(x, t)$. We consider the function $w$ in $(3.41)$ with $(\hat{x}, \hat{t})=(x, t)$. Then by (3.42) and by the Schauder estimates in Theorem 2.1,

$$
\sup _{Q_{1 / 2}}|Y w| \leq\|Y w\|_{C_{K}^{0, \alpha}\left(Q_{1 / 2}\right)} \leq \hat{c}_{6}
$$

and

$$
|w((y, s) \circ(0, h))-w(y, s)| \leq \hat{c}_{6}|h| .
$$

Therefore, setting $\delta=r^{2} h$, we have

$$
|u((\hat{x}, \hat{t}) \circ(0, \delta))-u((\hat{x}, \hat{t}))|=r^{1+\alpha}|w((y, s) \circ(0, h))-w(y, s)| \leq \hat{c}_{6} r^{1+\alpha}|h| \leq \hat{c}_{6}|\delta|^{\frac{1+\alpha}{2}} .
$$

By Remark 1.1 it then follows that $\|u\|_{C_{K}^{1, \alpha}\left(Q_{R}\right)}$ is bounded by a constant only dependent on $\alpha$, the operator $L$ and the data $f, g$ and $\psi$.

Proof of Theorem 1.7 We first note, as in the proof of Lemma 3.4, that it is not restrictive to consider $\psi \equiv 0$ and hence we assume that

$$
(u, g, f, \psi) \in \mathscr{P}_{2}\left(Q, \alpha, \mathbf{c}_{\alpha}, M_{1}, M_{2}, M_{3}\right),
$$

with $\psi \equiv 0$ and $M_{3}=0$. Let $R$ and $\mathscr{F}$ be as in the proof of Theorem 1.5 . We intend to prove that there exists $\hat{c}=\hat{c}\left(N, \lambda, \alpha, \mathbf{c}_{\alpha}, M_{1}, M_{2}\right)<\infty$ such that

$$
\|u\|_{S^{\infty}\left(Q_{R}\right)} \leq \hat{c} .
$$

For any $(\hat{x}, \hat{t}) \in Q_{R} \cap\{(x, t): u(x, t)>0\}$, we introduce

$$
\hat{r}=\hat{r}(\hat{x}, \hat{t})=\sup \left\{r: Q_{r}(\hat{x}, \hat{t}) \subset Q \cap\{(x, t): u(x, t)>0\}\right\} .
$$

As $\mathscr{F} \cap \partial_{P} Q_{\hat{r}}(\hat{x}, \hat{t}) \neq \emptyset$ (by the maximum principle) we see that there exists $(\tilde{x}, \tilde{t}) \in Q_{2 R} \cap \mathscr{F} \cap$ $\partial_{P} Q_{\hat{r}}(\hat{x}, \hat{t})$ such that $Q_{\hat{r}}(\hat{x}, \hat{t}) \subset Q_{\tilde{r}}(\tilde{x}, \tilde{t})$ for some $\tilde{r}>0$ such that $\hat{r} \leq \tilde{r} \leq c_{0} \hat{r}$, where $c_{0}$ is a suitable universal constant. Next using Lemma 3.7, we find

$$
|u(x, t)| \leq c r^{2} \quad \text { whenever }(x, t) \in Q_{r}(\tilde{x}, \tilde{t}) \cap Q \quad 0<r<\tilde{r} .
$$


We next define, for every $(x, t) \in Q, v(x, t)=\hat{r}^{-2} u^{\hat{r},(\hat{x}, \hat{t})}(x, t)$. Clearly, $v$ satisfies $L^{\hat{r},(\hat{x}, \hat{t})} v=f^{\hat{r},(\hat{x}, \hat{t})}$ in $Q$ and by using (3.48) we see that

$$
\|v\|_{L^{\infty}(Q)} \leq c, \quad\left\|f^{\hat{r},(\hat{x}, \hat{t})}\right\|_{C_{K}^{0, \alpha}(Q)} \leq M_{2}
$$

Finally, using Theorem 2.1 we can therefore conclude that

$$
\|v\|_{S^{\infty}\left(Q_{1 / 2}(\hat{x}, \hat{t})\right)} \leq\|v\|_{C_{K}^{2, \alpha}\left(Q_{1 / 2}(\hat{x}, \hat{t})\right)} \leq \hat{c} .
$$

where the constant $\hat{c}$ only depends on $N, \lambda, \alpha, \mathbf{c}_{\alpha}, M_{1}, M_{2}$ and $M_{3}$. Our claim (3.46) then follows from the above inequality. This completes the proof of Theorem 1.7 .

\section{References}

[1] E. Barucci, S. Polidoro, And V. Vespri, Some results on partial differential equations and Asian options, Math. Models Methods Appl. Sci., 11 (2001), pp. 475-497.

[2] A. Bensoussan, On the theory of option pricing, Acta Appl. Math., 2 (1984), pp. 139-158.

[3] A. Bonfiglioli, Taylor formula for homogenous groups and applications, to appear in Math. Z., (2008).

[4] L. Caffarelli, A. Petrosyan, and H. Shahgholian, Regularity of a free boundary in parabolic potential theory, J. Amer. Math. Soc., 17 (2004), pp. 827-869 (electronic).

[5] C. Cinti And S. Polidoro, Bounds on short cylinders and uniqueness results for degenerate Kolmogorov equations, preprint AMS Acta, (2008).

[6] G. Citti, A. Pascucci, And S. Polidoro, On the regularity of solutions to a nonlinear ultraparabolic equation arising in mathematical finance, Differential Integral Equations, 14 (2001), pp. 701-738.

[7] M. Di Francesco And A. Pascucci, On the complete model with stochastic volatility by Hobson and Rogers, Proc. R. Soc. Lond. Ser. A Math. Phys. Eng. Sci., 460 (2004), pp. 3327-3338.

[8] - On a class of degenerate parabolic equations of Kolmogorov type, AMRX Appl. Math. Res. Express, (2005), pp. 77-116.

[9] M. Di Francesco, A. Pascucci, And S. Polidoro, The obstacle problem for a class of hypoelliptic ultraparabolic equations, Proc. R. Soc. Lond. Ser. A Math. Phys. Eng. Sci., 464 (2008), pp. 155-176.

[10] M. Di Francesco And S. Polidoro, Schauder estimates, Harnack inequality and Gaussian lower bound for Kolmogorov-type operators in non-divergence form, Adv. Differential Equations, 11 (2006), pp. 1261-1320.

[11] P. Foschi And A. Pascucci, Path dependent volatility, Decis. Econ. Finance, 31 (2008), pp. 13-32. 
[12] A. Friedman, Variational principles and free-boundary problems, Robert E. Krieger Publishing Co. Inc., Malabar, FL, second ed., 1988.

[13] D. G. Hobson And L. C. G. Rogers, Complete models with stochastic volatility, Math. Finance, 8 (1998), pp. 27-48.

[14] L. Hörmander, Hypoelliptic second order differential equations, Acta Math., 119 (1967), pp. 147-171.

[15] P. Jaillet, D. Lamberton, and B. Lapeyre, Variational inequalities and the pricing of American options, Acta Appl. Math., 21 (1990), pp. 263-289.

[16] I. Karatzas, On the pricing of American options, Appl. Math. Optim., 17 (1988), pp. 3760.

[17] D. Lamberton, American options , in Statistics in finance, Hand, David J. (ed.) and Jacka, Saul D. (ed.) Arnold Applications of Statistics Series. London: Arnold. x, 340 p., 1998.

[18] E. Lanconelli and S. Polidoro, On a class of hypoelliptic evolution operators, Rend. Sem. Mat. Univ. Politec. Torino, 52 (1994), pp. 29-63.

[19] A. Nagel, E. M. Stein, and S. Wainger, Balls and metrics defined by vector fields. I. Basic properties, Acta Math., 155 (1985), pp. 103-147.

[20] A. PascuCcI, Free boundary and optimal stopping problems for American Asian options, Finance Stoch., 12 (2008), pp. 21-41.

[21] A. Petrosyan and H. Shahgholian, Parabolic obstacle problems applied to finance, in Recent developments in nonlinear partial differential equations, vol. 439 of Contemp. Math., Amer. Math. Soc., Providence, RI, 2007, pp. 117-133.

[22] P. VAn Moerbeke, Optimal stopping and free boundary problems, Rocky Mountain J. Math., 4 (1974), pp. 539-578. Papers arising from a Conference on Stochastic Differential Equations (Univ. Alberta, Edmonton, Alta., 1972).

[23] — An optimal stopping problem with linear reward, Acta Math., 132 (1974), pp. 111-151. 